\title{
The Gaia DR2 halo white dwarf population: the luminosity function, mass distribution and its star formation history
}

\author{
Santiago Torres ${ }^{1,2},{ }^{\star}$ Alberto Rebassa-Mansergas ${ }^{1,2}$, María E. Camisassa ${ }^{1,3,4}$ and \\ Roberto Raddi ${ }^{1}$ \\ ${ }^{1}$ Departament de Física, Universitat Politècnica de Catalunya, c/Esteve Terrades 5, 08860 Castelldefels, Spain \\ ${ }^{2}$ Institute for Space Studies of Catalonia, c/Gran Capita 2-4, Edif. Nexus 104, 08034 Barcelona, Spain \\ ${ }^{3}$ Facultad de Ciencias Astronómicas y Geofísicas, Universidad Nacional de La Plata, Paseo del Bosque s/n, 1900 La Plata, Argentina \\ ${ }^{4}$ Instituto de Astrofísica de La Plata, UNLP-CONICET, Paseo del Bosque s/n, 1900 La Plata, Argentina
}

Accepted XXX. Received YYY; in original form ZZZ

\section{ABSTRACT}

We analyze the volume-limited nearly complete $100 \mathrm{pc}$ sample of 95 halo white dwarf candidates identified by the second data : release of Gaia. Based on a detailed population synthesis model, we apply a method that relies on Gaia astrometry and photometry , to accurately derive the individual white dwarf parameters (mass, radius, effective temperature, bolometric luminosity and age). ' This method is tested with 25 white dwarfs of our sample for which we took optical spectra and performed spectroscopic analysis. - We build and analyse the halo white dwarf luminosity function, for which we find for the first time possible evidences of the cut-off at its faintest end, leading to an age estimate of $\simeq 12 \pm 0.5 \mathrm{Gyr}$. The mass distribution of the sample peaks at $0.589 M_{\odot}$, ' with $71 \%$ of the white dwarf masses below $0.6 M_{\odot}$ and just two massive white dwarfs of more than $0.8 M_{\odot}$. From the age ', distribution we find three white dwarfs with total ages above $12 \mathrm{Gyr}$, of which J1312-4728 is the oldest white dwarf known with ' an age of $12.41 \pm 0.22 \mathrm{Gyr}$. We prove that the star formation history is mainly characterised by a burst of star formation that ' occurred from 10 to $12 \mathrm{Gyr}$ in the past, but extended up to $8 \mathrm{Gyr}$. We also find that the peak of the star formation history is ' centered at around $11 \mathrm{Gyr}$, which is compatible with the current age of the Gaia-Enceladus encounter. Finally, $13 \%$ of our halo . sample is contaminated by high-speed young objects (total age $<7 \mathrm{Gyr}$ ). The origin of these white dwarfs is unclear but their age ' distribution may be compatible with the encounter with the Sagittarius galaxy.

Key words: stars: white dwarfs - Galaxy: stellar content - stars: luminosity function, mass function - Galaxy: halo

White dwarfs are long-lived objects whose evolutionary characteristics are reasonably well understood (e.g. Althaus et al. 2010, and references therein). They represent the vast majority of low- and intermediate-mass stars remnants. Thus, their ensemble properties carry valuable information about the past history and evolution of the different components of the Galaxy. In particular, white dwarfs are reliable cosmochronometers and, consequently, they have been used for studying several Galactic populations. As illustrative examples of this, we can mention the analysis of the Galactic thin and thick disk (e.g. García-Berro et al. 1999; Torres et al. 2002; Rowell \& Hambly 2011; Kilic et al. 2017), the halo (e.g. Mochkovitch et al. 1990; Isern et al. 1998; García-Berro et al. 2004; Cojocaru et al. 2015) the bulge (e.g Calamida et al. 2014; Torres et al. 2018) or precise studies of Galactic open and globular clusters (e.g. García-Berro et al. 2010; Jeffery et al. 2011; Hansen et al. 2013; Torres et al. 2015).

Regarding the population of white dwarfs in the Galactic stellar halo, it has been the focus of attention in this field since the first observational and theoretical studies (Mochkovitch et al. 1990; Liebert et al. 1989; Isern et al. 1998). Shortly after, and since the MACHO collaboration experiment for the microlensing detection (e.g. Alcock et al. 2000), halo white dwarfs have been suggested as natural candidates to contribute to the dark matter content of the Galaxy (e.g. Oppenheimer et al. 2001). An intense debate arose on this issue, although later studies demonstrated that the white dwarf contribution to dark matter was rather limited (e.g. Torres et al. 2002; Flynn et al. 2003; García-Berro et al. 2004; Kilic et al. 2004; Bergeron et al. 2005). In any case, the search for white dwarfs in the Galactic halo has proven to be a difficult endeavour. In this sense, the intrinsic faintness and the low space density of halo white dwarfs, along with their high surface gravity, which erases any trace on metal content from their atmospheres and disables accurate radial velocity measurements due to the broadening of the Balmer lines, are some of the main factors that have hindered the discovery of suitable candidates. Consequently, during decades, the identification of halo members has relied on the search of cool objects in high proper motion surveys (e.g. Monet et al. 2000; Nelson et al. 2002; Hall et al. 2008; Catalán et al. 2012; Munn et al. 2016). It was not until the ESO SNIa Progenitor surveY (SPY) project (see Napiwotzki et al. 2020, and references therein) that radial velocities were measured for the first time with enough precision to recover the 3D kinematics of white dwarfs. That permitted to identify high eccentric retrograde orbits as strongly indicators of their belonging to the halo population (Pauli et al. 2006). Although the number of halo members was

^ E-mail: santiago.torres@upc.edu 
sparse, an estimate of the age of the inner halo has been possible ( 11.4 Gyr; Kalirai 2012).

A key ingredient of these studies is the white dwarf luminosity function. Defined as the number of white dwarfs per bolometric magnitude unit and cubic parsec, the white dwarf luminosity function is a valuable tool to derive the age, history and evolution of the components of our Galaxy. Moreover, the luminosity function holds all the information about the cooling process of the white dwarfs, being then an excellent tool for testing the physics of evolutionary models (see García-Berro \& Oswalt 2016, for a comprehensive review about these issues). However, the effectiveness of the white dwarf luminosity function requires the existence of a complete, welldefined volume sample. In this sense, the lack of accurate distances previous to the Gaia era, joint to the selection biases inherited from magnitude-limited samples, have hampered the achievement of more conclusive results in such an elusive sample as the halo white dwarf population.

The first observational halo white dwarf luminosity function (Liebert et al. 1989) contained only five objects, and no more than 20 were proposed as halo candidates through a neural network artificial intelligent classification algorithm (Torres et al. 1998). Based on the SuperCOSMOS Sky Survey observations towards the southern Galactic Cap, Oppenheimer et al. (2001) identified 38 hightangential velocity white dwarfs, from which they deduced that white dwarfs may represent $2 \%$ of the local dark matter density. An intense debate began in this regard, mainly based on the difficulty of unequivocally identifying halo members. Currently, several halo white dwarf luminosity functions have been proposed (e.g. Rowell \& Hambly 2011; Munn et al. 2016; Lam et al. 2018). These samples, based on reduced-proper motion surveys, are magnitude limited. Thus, different statistical approaches, starting from the well-known maximum volume estimator method, have been applied in order to correct the observational biases of the intrinsic incompleteness of magnitude limited samples. Parallel to the search for statistical significant halo white dwarf samples, a major effort has been devoted to identify individual halo white dwarf members. The list includes from hot and warm objects up to cool and ultracool white dwarfs (e.g. Hambly et al. 1997; Ibata et al. 2000; Pauli et al. 2006; Kalirai 2012; Kawka \& Vennes 2012; Gianninas et al. 2015; Si et al. 2017).

However, as stated before, achieving a statistical complete sample of halo white dwarfs has become an arduous task, at least during the pre-Gaia era. A first estimate of the number of halo white dwarfs showed that up to magnitude $G<20$, around 85 objects within $100 \mathrm{pc}$ and $\sim 500$ objects within $400 \mathrm{pc}$ are expected to be accessible by Gaia (Torres et al. 2005). Since Gaia Data Release 2 was published only a few studies of the Galactic halo white dwarf population have been published. In Kilic et al. (2018), a sample of 142 objects inconsistent with disk kinematics has been analyzed. In particular, an age estimate has been provided for many of the members of the sample, obtaining, from the coolest white dwarfs, an age estimate of the inner halo of $10.9 \mathrm{Gyr}$. However, the sample, which contains objects as far as $540 \mathrm{pc}$, is far from being complete. On the other hand, Torres et al. (2019a) focused on the search of halo white dwarfs in the near-complete sample within $100 \mathrm{pc}$ from the Sun. With the help of artificial intelligence algorithms, Torres et al. (2019a) categorized the membership of white dwarfs in the different Galactic components, identifying the largest volume-complete halo white dwarf sample, consisting in 95 stars.

A renewed interest on the halo population has appeared since the astrometric Gaia mission has provided accurate parallax and proper motion measurements for approximately 1.4 billion stars of our Galaxy (Gaia Collaboration et al. 2018). In the new Gaia-Enceladus paradigm, a major impact event took place in our Galaxy around 10.5-11.5 Gyr in the past, being at the origin of the formation of the thick disk and inner stellar halo (Helmi et al. 2018; Gallart et al. 2019). The precise age, intensity and effects of this past event on the kinematics and properties of the stars in the solar neighborhood are not yet well understood.

In this paper, we analyze the halo white dwarf sample identified in Torres et al. (2019a). In particular, we obtain the halo white dwarf luminosity function, the mass distribution of the sample and an estimate of its star formation history. To achieve this goal, we complement our analysis with spectroscopic observation of 27 stars of the sample. The stellar parameters of each white dwarf (i.e. luminosity, age and mass) are obtained from a robust method which makes use of Gaia photometry and astrometry together with a detailed population synthesis code, based on Monte Carlo techniques and which incorporates the most up-to-date evolutionary sequences of white dwarfs. Finally, it is important to emphasise that we use the term halo to describe that sample whose characteristics are different from the average thin or thick disk white dwarf sample. If these objects really belong to an ancient halo, inner spheroid or are the remnants of a major merger event, it is something that will be analyzed along the paper.

The paper is organized as follows. In the first section we describe our halo white dwarf sample. That includes how we identified the halo candidates, the fundamental parameters that were derived from those spectroscopically observed and the main characteristics of our population synthesis modeling. In the second section we present our strategy and testing for deriving the fundamental parameters extended to the whole halo white dwarf sample. Then, the halo white dwarf luminosity function, mass distribution and star formation history are presented. Finally, in Section 3 we analyze the results achieved and summarize them in the concluding remarks.

\section{THE HALO WHITE DWARF SAMPLE}

\subsection{Identification of halo white dwarfs}

Gaia Data Release 2 has provided a wealth of unprecedented information concerning the Galactic white dwarf population (Jiménez-Esteban et al. 2018; Gentile Fusillo et al. 2019). The high astrometric accuracy and the photometry provided by Gaia has allowed us to build a clean color-magnitude diagram to select a large sample of white dwarf candidates. With the aid of a detailed population synthesis simulator, we analysed the main properties of the white dwarf population available from Gaia (Jiménez-Esteban et al. 2018). In this study, we showed that the largest and most complete sample of white dwarfs available by Gaia is up to $100 \mathrm{pc}$ from the Sun. For larger distances, the completeness of the sample decays dramatically and biases and magnitude-selection effects begin to grow in importance.

With the aid of advanced intelligent algorithms, we studied the main properties of the Gaia $100 \mathrm{pc}$ white sample (Torres et al. 2019a). In particular, we have been able to disentangle the white dwarf populations from the different components of the Galaxy, i.e., thin and thick disk, and halo. The artificial intelligent method used consisted in a supervised method based on Random Forest techniques. This Random Forest algorithm was applied to an 8-dimensional space formed by equatorial coordinates, parallax, proper motion components and photometric magnitudes. This 8-dimensional space permits the algorithm to maximize the information in order to classify its different components. Our results showed that the algorithm presents an accuracy of $85.3 \%$. In particular, our analysis indicates that $80 \%$ 

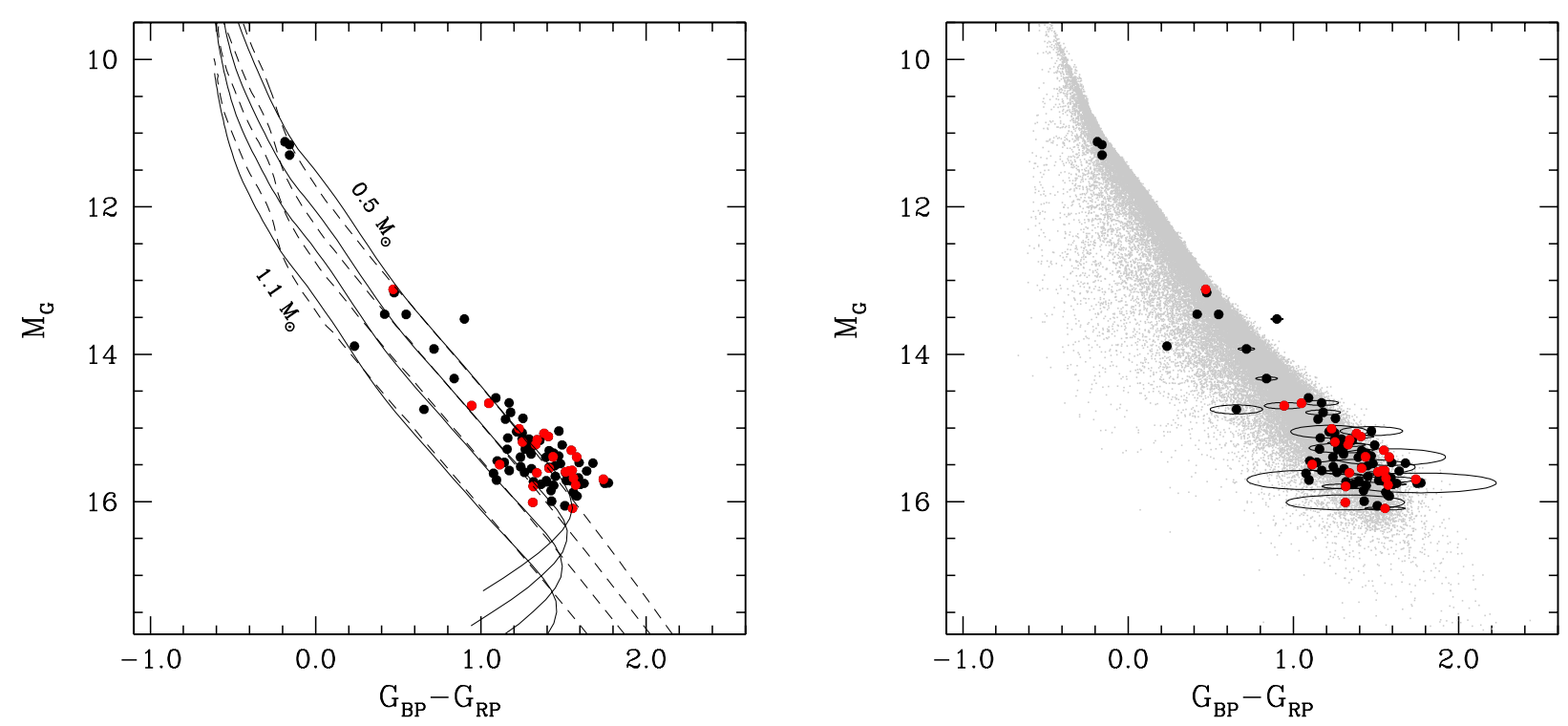

Figure 1. Gaia HR-diagram for our halo white dwarf sample within $100 \mathrm{pc}$. Marked as red circles are those white dwarfs for which we obtained optical spectra. In the left panel we plot the theoretical cooling tracks for DA (solid lines) and DB (dashed lines) white dwarfs for different masses ranging from 0.5 to $1.1 M_{\odot}$ in steps of $0.2 M_{\odot}$. In the right panel a typical simulation (gray dots) taking into account photometric and astrometric errors is shown. For illustrative purposes and for the sake of clarity we only plot some $1 \sigma$ ellipses. See text for details.

of possible halo white dwarfs in the $100 \mathrm{pc}$ sample are expected to be correctly identify, and only a low $5 \%$ contamination is expected. These scores are higher than any other obtained by tangential velocity cuts, Toomre diagram criteria or reduced proper motion criteria usually applied for selecting the different kinematic populations. Moreover, practically all white dwarfs within $100 \mathrm{pc}$ that were previously identified in the literature as halo members are also included in our sample. Thus, the sample found represents the most complete and largest volume-limited sample of the halo white dwarf population to date. However, these facts do not exclude the need to perform a detailed analysis of the completeness of the sample, in particular, for the faint region of the sample. This is done in Section 2.2.

The sample consists of 95 halo white dwarfs candidates representing an old and high velocity population. Its space density is $(4.8 \pm 0.4) \times 10^{-5} \mathrm{pc}^{-3}$, accounting for $1 \%$ of the whole white dwarf population within $100 \mathrm{pc}$. A complete list of the 95 halo members with their Gaia source ID, coordinates and main parameters is shown in Table 3 of Torres et al. (2019a). In Figure 1 we plot our 95 halo candidates in the Gaia Hertzsprung-Russell (HR) diagram. For illustrative purposes we also plot the cooling tracks belonging to hydrogen-rich and pure helium atmosphere models for different masses. As shown in Fig. 1, many objects lie near the blue-hook of the hydrogen-rich atmosphere tracks, thus representing cold and old objects. At first sight, however, it seems to reveal that many of these objects have low masses, $\leq 0.5 M_{\odot}$, even when compared with $\mathrm{He}-$ pure atmosphere tracks. This effect was analyzed by Bergeron et al. (2019) claiming that probably most of the possible non-DA objects in this region are DA or a large fraction of unresolved double degenerates populates this region. However, we shall see in Section 1.3 that this apparent shift towards lower masses is nicely resolved when taking into account photometric and astrometric errors in the observed data.

\subsection{Spectroscopic observation of halo white dwarfs}

We obtained low-resolution spectra for 27 of our 95 halo white dwarf candidates with the Very Large Telescope UT1 equipped with the FOcal Reducer/low-dispersion Spectrograph (FORS2) (Appenzeller et al. 1998). We used the GRIS_1200+97 grism and the 1 " slit width, which resulted in spectra covering the $\simeq 3800$ $5200 \AA$ wavelength range at a resolving power of $R \simeq 1400$. We used the Pamela software (Marsh 1989) to subtract the sky contribution and to extract the one dimensional spectra. The data were then wavelength and flux calibrated using arc lamps and flux standard stars taken at the same time of the observations within the MOLLY package $^{1}$. Visual inspection of the flux-calibrated spectra revealed that 24 targets were DC white dwarfs, a result which is not surprising given that these objects are expected to be very old and cold. The remaining three white dwarfs were classified as two DQs (J0248-3001 and J1159-4629) and one DA (J0148-1712). The Gaia source ID, short name, Gaia absolute magnitude $M_{\mathrm{G}}$, colour $G_{\mathrm{BP}}-G_{\mathrm{RP}}$ and spectral type of the $24 \mathrm{DC}+1 \mathrm{DA}$ observed white dwarfs are, respectively, provided in the first five columns of Table 1.

In order to derive the stellar parameters of the white dwarfs with available spectra we used the fitting routine of Rebassa-Mansergas et al. (2007). Briefly, this procedure uses a grid of model spectra (Koester 2010) ${ }^{2}$ to fit both the continuum and the normalised Balmer lines of the spectra to derive the effective temperatures and surface gravities. By adopting then a white dwarf

\footnotetext{
1 Tom Marsh's MOLLY package is available at http://deneb.astro.warwick.ac.uk/phsaap/software

2 The grid includes 1260 spectra of effective temperatures between 3000 and $40,000 \mathrm{~K}$ in steps of $250 \mathrm{~K}$ up to $20,000 \mathrm{~K}$, steps of $1000 \mathrm{~K}$ up to $30,000 \mathrm{~K}$ and steps of $2000 \mathrm{~K}$ up to $40,000 \mathrm{~K}$; and surface gravities between 6 and $9.5 \mathrm{dex}$ in steps of 0.25 dex for each effective temperature. Prior to the fit, the model spectra were folded at the resolving power of the observed spectra.
} 

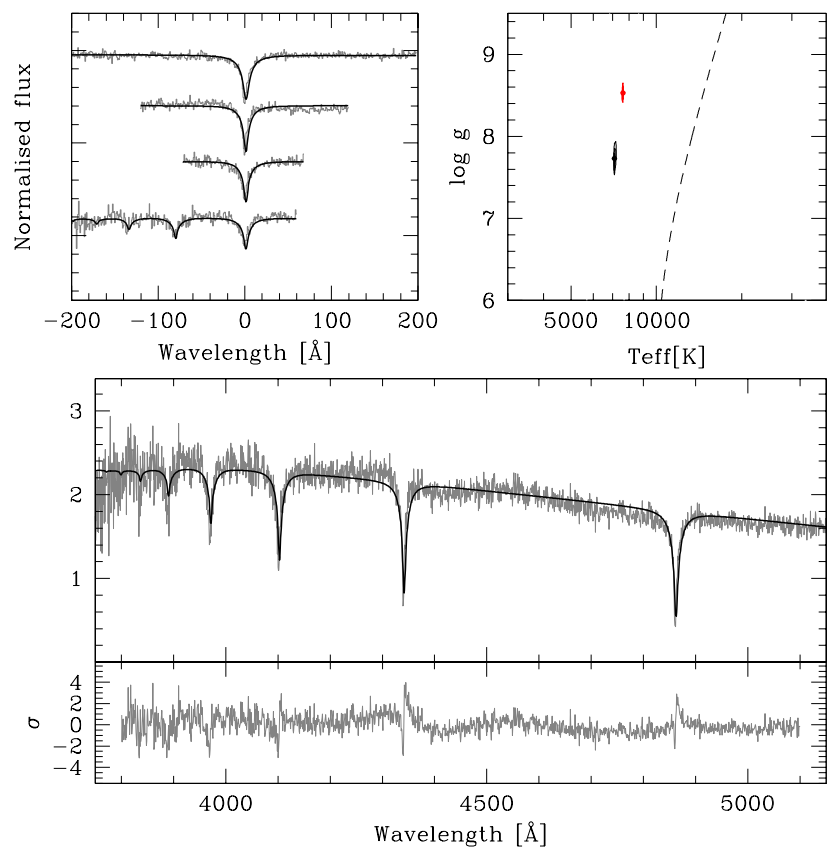

Figure 2. Spectral model fit to the DA white dwarf J0148-1712. Top left panel: best-fit (black lines) to the normalised Balmer line profiles sampled by the FORS2 spectrum (gray lines). Top right panel: 3, 5, and $10 \sigma \chi^{2}$ contour plots in the $\mathrm{T}_{\mathrm{eff}}-\log g$ plane. The black contours refer to the best line profile fit, the red contours to the fit of the whole spectrum. The dashed line indicates the occurrence of maximum $\mathrm{H} \beta$ equivalent width. The best "hot" (not visible in the figure) and "cold" line profile solutions are indicated by black dots, the best fit to the whole spectrum is indicated by a red dot. Bottom panels: the white dwarf spectrum (gray line) along with the best-fit white dwarf model (black line) (top) and the residuals of the fit (gray line, bottom).

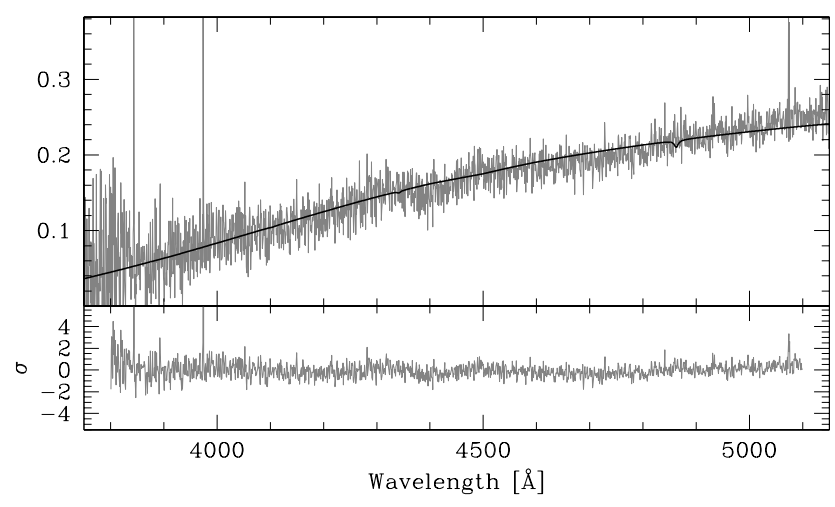

Figure 3. Spectral model fit to the DC white dwarf J2117-4156. Top panel: the white dwarf spectrum (gray line) along with the best-fit white dwarf model (black line). Bottom panel: the residuals of the fit (gray line).

mass-radius relation (e.g. Camisassa et al. 2016), the mass and radius (hence bolometric luminosity since the temperature is known) are also obtained. This routine is only valid if the white dwarfs are hydrogen-rich DAs. In this case, the fit to the continuum is only used to differentiate between the so-called hot and cold solutions obtained from the Balmer line fitting. The fit to our only DA halo white dwarf candidate with available FORS2 spectrum is shown in Figure2. However, for our DC white dwarfs, which are the vast majority of the spectroscopically observed sample, we could only rely on the fits to the continuum since these objects are absent of any lines. Thus, only the effective temperatures were obtained in these cases. A fit to one of our DC white dwarf halo candidates can be seen in Figure3.

We note that another possibility exists for deriving the white dwarf radii of our DC white dwarfs by using the flux scaling factors between the observed spectra and the best fit models, since the distances are known from the Gaia parallaxes. Hence, a spectroscopic bolometric luminosity could be obtained from the effective temperatures and radii via the Stefan-Boltzmann equation. We performed a first attempt to follow this procedure by first re-scaling the observed spectra to the Gaia fluxes (and PanStarrs fluxes when available too) of our stars.

The radii, effective temperatures and bolometric luminosities thus derived for our DA and the rest of DC halo white dwarf candidates are shown, respectively, in the last three columns of Table 1. In Section 2.1 we discuss the validity of the spectroscopic parameter values thus obtained.

\subsection{The synthetic halo white dwarf population}

We complement our analysis of the 95 halo white dwarfs candidates with the aid of a detailed population synthesis code. Our code, based on Monte Carlo techniques, has been widely used in the study of the white dwarf population of the different Galactic components, i.e., disk, halo and bulge, as well as in globular and open clusters (e.g. García-Berro et al. 1999; García-Berro et al. 2004, 2010; Torres et al. 2001; Torres et al. 2002, 2015; Torres \& García-Berro 2016; Torres et al. 2018). Here we will only mention the main ingredients of our halo simulation, while a thorough description of the code, as well as its Gaia performances, can be found in Torres et al. (2005); Jiménez-Esteban et al. (2018); Torres et al. (2019a).

The main objective of our halo white dwarf simulation is to overpopulate the entire white dwarf region of the HR-diagram space. Synthetic white dwarfs will be used then to extract the physical parameters at the HR-diagram loci where observed white dwarfs have been found. For this reason, it is not necessary to take into account a meaningful star formation history, nor an exact age of the halo population. Consequently, we just adopt a constant star formation rate and we generate stars with an upper-limiting age fixed at the age of the Universe of 13.7 Gyr (Planck Collaboration et al. 2016). Stars generated are drawn form a Salpeter initial mass function with a standard slope value of $\alpha=-2.35$. We considered that white dwarfs are formed only through single evolution. Additionally, as done in the halo white dwarf analysis by Kilic et al. (2018), a constant metallicity value of $[\mathrm{Fe} / \mathrm{H}]=-1.5$ is adopted. Main-sequence lifetimes are drawn from BaSTI models (Hidalgo et al. 2018) and the semiempirical initial-to-final mass relationship of Catalán et al. (2008) is applied. White dwarf cooling times are derived from a complete set of cooling sequences, which encompass the full range of masses (Althaus et al. 2015; Camisassa et al. 2017, 2019). It is worth noting that these cooling sequences are specifically calculated for the adopted metallicity and are the result of the full previous progenitor evolution, starting at the zero-age main sequence, all the way through central hydrogen and helium burning, thermally pulsing asymptotic giant branch (AGB) and post-AGB phases. The lifetime of the white dwarf progenitors along with the initial-to-final mass relationship obtained this way are in completely agreement with those input models previously stated. This fact guarantees us the use of a coherent set of evolutionary sequences. Hydrogen-rich and hydrogen-deficient atmosphere models are generated according to the canonical distribution of $80 \%$ and $20 \%$, respectively. For the spatial distribution an isothermal model is adopted, which is practically equivalent to an isotropic distribution for the local $100 \mathrm{pc}$ neighborhood. Magnitudes 
Table 1. Spectroscopic determinations of the parameters of the halo white dwarf candidates for which the spectrum is available. The stellar parameters derived, i.e., radius, effective temperature and bolometric luminosity, are shown, respectively, in the last three columns.

\begin{tabular}{|c|c|c|c|c|c|c|c|}
\hline $\begin{array}{c}\text { Gaia } \\
\text { source ID }\end{array}$ & $\begin{array}{l}\text { Short } \\
\text { name }\end{array}$ & $\begin{array}{c}M_{\mathrm{G}} \\
(\mathrm{mag})\end{array}$ & $\begin{array}{c}G_{\mathrm{BP}}-G_{\mathrm{RP}} \\
\quad(\mathrm{mag})\end{array}$ & $\begin{array}{l}\text { Spectral } \\
\text { type }\end{array}$ & $\begin{array}{l}\text { Radius } \\
\left(R_{\odot} / 10^{3}\right)\end{array}$ & $\begin{array}{l}T_{\text {eff }} \\
(K)\end{array}$ & $\begin{array}{l}\text { Luminosity } \\
\left(L_{\odot} / 10^{5}\right)\end{array}$ \\
\hline 5042228731477861888 & J0129-2257 & 15.55 & 1.41 & DC & $11.76 \pm 0.97$ & $4094 \pm 100$ & $3.49 \pm 0.83$ \\
\hline 5142197118950177280 & J0148-1712 & 13.12 & 0.47 & DA & $15.90 \pm 1.09$ & $7144 \pm 50$ & $59.15 \pm 10.56$ \\
\hline 2490975272405858048 & J0205-0517 & 15.59 & 1.53 & DC & $10.64 \pm 0.69$ & $4094 \pm 100$ & $2.86 \pm 0.58$ \\
\hline 4616895783694397184 & J0237-8445 & 15.77 & 1.57 & DC & $13.25 \pm 2.31$ & $3955 \pm 100$ & $3.85 \pm 1.64$ \\
\hline 5188044687948351872 & J0301-0044 & 15.30 & 1.55 & DC & $10.89 \pm 1.05$ & $4287 \pm 100$ & $3.60 \pm 0.95$ \\
\hline 4862884499360563968 & J0340-3301 & 15.79 & 1.32 & DC & $10.58 \pm 0.89$ & $4238 \pm 100$ & $3.24 \pm 0.77$ \\
\hline 3249657094642979840 & J0342-0344 & 15.50 & 1.11 & DC & $10.90 \pm 0.91$ & $4541 \pm 100$ & $4.54 \pm 1.05$ \\
\hline 2989049057626796416 & J0518-1155 & 15.70 & 1.74 & DC & $9.45 \pm 1.02$ & $4001 \pm 100$ & $2.05 \pm 0.60$ \\
\hline 5228861484450843648 & J1049-7400 & 16.09 & 1.55 & DC & $8.22 \pm 0.87$ & $4047 \pm 100$ & $1.63 \pm 0.47$ \\
\hline 3801499128765222400 & J1053-0307 & 15.39 & 1.44 & DC & $11.19 \pm 0.98$ & $4141 \pm 100$ & $3.31 \pm 0.82$ \\
\hline 5348874243767794304 & $\mathrm{~J} 1123-5150$ & 15.61 & 1.34 & DC & $11.89 \pm 1.19$ & $4865 \pm 100$ & $2.83 \pm 1.87$ \\
\hline 5377861317357370240 & J1159-4630 & 15.19 & 0.42 & DC & $8.32 \pm 0.74$ & $4489 \pm 100$ & $2.52 \pm 0.62$ \\
\hline 6085402414245451520 & $\mathrm{~J} 1312-4728$ & 15.58 & 1.54 & DC & $10.58 \pm 0.79$ & $4094 \pm 100$ & $2.83 \pm 0.63$ \\
\hline 6165095738576250624 & J1342-3415 & 14.66 & 1.05 & DC & $10.88 \pm 0.85$ & $5095 \pm 100$ & $7.16 \pm 1.54$ \\
\hline 5824436284328653312 & J1517-6645 & 16.01 & 1.31 & DC & $9.71 \pm 1.33$ & $3865 \pm 100$ & $1.89 \pm 0.67$ \\
\hline 6007140379167609984 & $\mathrm{~J} 1518-3803$ & 15.68 & 1.56 & DC & $10.51 \pm 1.11$ & $4189 \pm 100$ & $3.05 \pm 0.87$ \\
\hline 5827557213731539328 & J1539-6124 & 15.23 & 1.33 & DC & $11.62 \pm 0.89$ & $4336 \pm 100$ & $4.29 \pm 0.96$ \\
\hline 5817295536128445568 & J1707-6319 & 15.12 & 1.41 & DC & $11.25 \pm 0.94$ & $4437 \pm 100$ & $4.40 \pm 1.04$ \\
\hline 6647162730439433984 & J1936-4913 & 15.60 & 1.51 & DC & $10.77 \pm 0.99$ & $4189 \pm 100$ & $3.21 \pm 0.82$ \\
\hline 6471523921227261056 & $\mathrm{~J} 2042-5218$ & 14.70 & 0.94 & DC & $11.65 \pm 0.86$ & $4755 \pm 100$ & $6.23 \pm 1.31$ \\
\hline 6580458035746362496 & $\mathrm{~J} 2117-4156$ & 15.16 & 1.34 & DC & $11.80 \pm 0.84$ & $4437 \pm 100$ & $4.85 \pm 1.02$ \\
\hline 6580551872194787968 & J2129-0034 & 15.39 & 1.58 & DC & $12.60 \pm 1.53$ & $4287 \pm 100$ & $4.81 \pm 1.51$ \\
\hline 2687584757658775424 & $\mathrm{~J} 2230-7515$ & 15.58 & 1.55 & DC & $10.62 \pm 0.82$ & $4141 \pm 100$ & $2.98 \pm 0.67$ \\
\hline 6357629089412187648 & J2319-0613 & 15.07 & 1.38 & DC & $7.05 \pm 0.74$ & $4979 \pm 100$ & $2.75 \pm 0.74$ \\
\hline 2631967439437024384 & J2349-0124 & 15.01 & 1.23 & DC & $13.41 \pm 1.08$ & $4287 \pm 100$ & $5.45 \pm 1.26$ \\
\hline
\end{tabular}

are interpolated in the corresponding cooling sequences and calculated in the Gaia filters (R. Rohrmann's private communication) using the appropriate atmosphere models. Finally, photometric and astrometric errors are added following Gaia's performance ${ }^{3}$.

A representative HR-diagram of our simulated halo white dwarf sample is presented in the right panel of Fig. 1. The number of objects generated is large enough to ensure statistical significance in all the regions around the observed objects. As it can be seen in Fig. 1 , the synthetic sample populates all the regions where our 95 halo white dwarf candidates are located. Only one object (J0055+3847) is outside of the simulated space, and this is probably because it is either a He-core white dwarf or a double degenerate system. It is also worth noting that those objects previously discussed in Section 1.1 as cold and abnormally shifted in the HR-diagram towards very low masses are now naturally recovered in the simulated space, once photometric and astrometric errors are taken into account.

\section{RESULTS}

\subsection{Methodology and testing}

The population synthesis sample described in the previous section allows us to derive the representative white dwarf parameters at each locus of the HR-diagram by just taking into account the Gaia photometric and astrometric errors. For each of the 95 objects of our halo sample we first derive the theoretical errors in $G$ magnitude and $G_{\mathrm{BP}}-G_{\mathrm{RP}}$ colour, $\sigma_{G}$ and $\sigma_{G_{\mathrm{BP}}-G_{\mathrm{RP}}}$, respectively. We build then a

\footnotetext{
${ }^{3}$ http://www.cosmos.esa.int/web/gaia/science-performance
}

region centered in the location of each object in the HR-diagram that contains all the synthetic stars within $1 \sigma$. The ellipses thus formed are shown in the right panel of Fig. 1. For the sake of clarity we only plot some of the most representatives. The $G$-magnitude error is mainly due to the photometric error in the $G$ filter, given that the astrometric error in parallax is practically negligible. On the contrary, the error in $G_{\mathrm{BP}}-G_{\mathrm{RP}}$ colour is the addition of the photometric errors from $G_{\mathrm{BP}}$ and $G_{\mathrm{RP}}$ filters. Thus, in general terms, the size of the $1 \sigma$ ellipses increases for dimmer objects, being the error in the absolute $G$ magnitude substantially smaller than the error in colour.

For each of the $1 \sigma$ ellipses we obtain the average luminosity, mass, age, effective temperature and radius of all synthetic white dwarfs within it. We can now compare the photometric parameters thus obtained with the spectroscopic ones derived for the 25 observed objects presented in Section 1.2. The results are plotted in Figure 4. On the top panels we show the comparison between the photometric versus the spectroscopic effective temperature (top left panel) and white dwarf radius (top right panel). The agreement in effective temperatures is good, since most of the objects are within the $1 \sigma$ errors. However, that is not the case for the white dwarf radii. Those obtained from the spectroscopic analysis are systematically lower than those obtained photometrically. This effect is most likely due to intrinsic errors when applying the flux calibration to our FORS2 spectra, which directly affect the radii measurements through the flux scaling factors.

Consequently, the luminosity thus obtained from the spectroscopic method suffers from this imprecision. In the bottom left panel of Fig. 4 we compare the photometric versus the spectroscopic luminosity for the objects in which the spectrum is available. Photometric luminosities are shifted towards larger (brighter) luminosities than those 

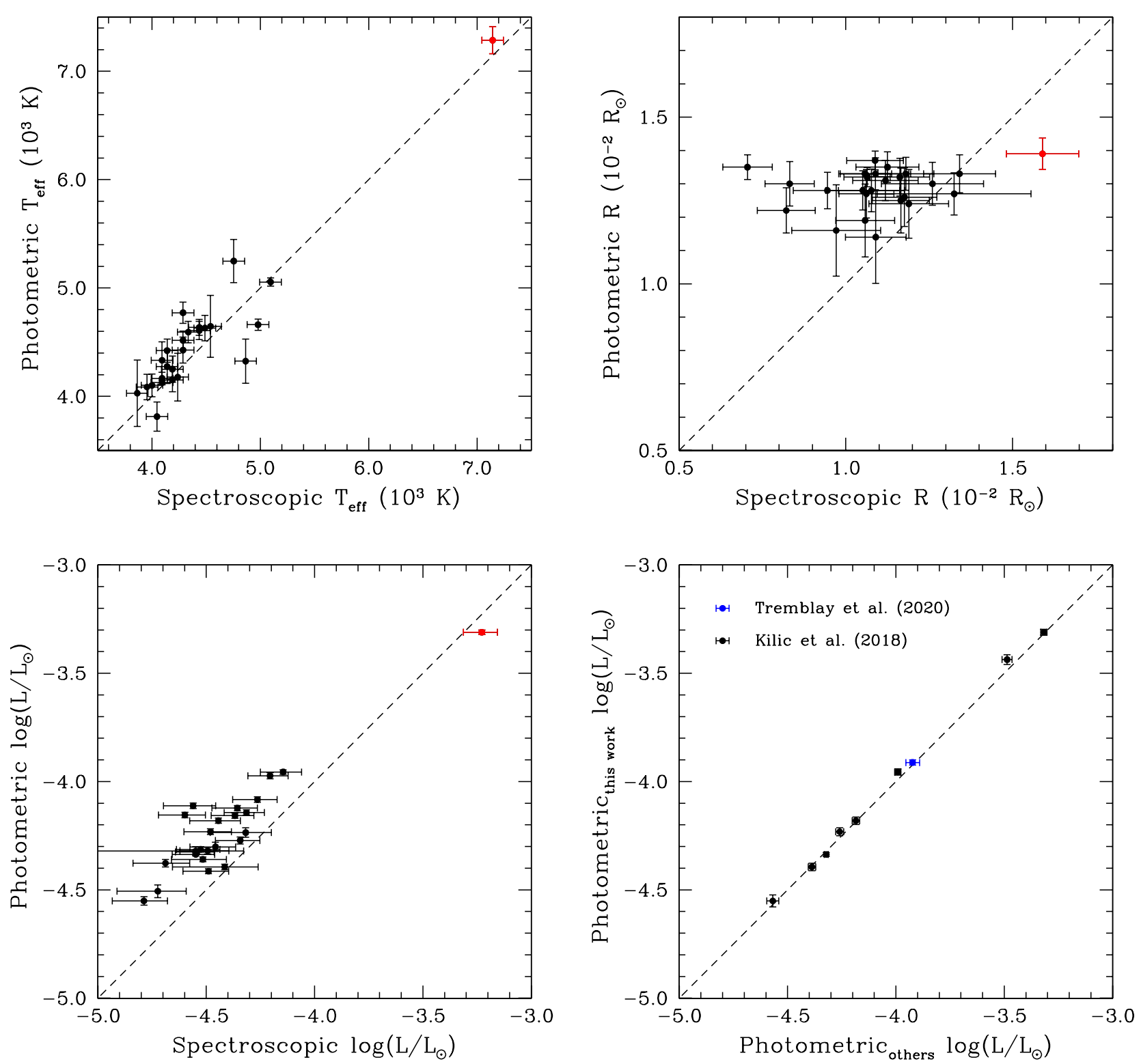

Figure 4. Spectroscopic effective temperatures (top left panel), radii (top right panel) and luminosities (bottom left panel) for the 24 DC (black dots) and one DA (red dot) observed objects (see Section 1.2) compared to the respective photometric parameters derived as the average value within $1 \sigma$ ellipses. Also shown (bottom right panel) is the photometric luminosity obtained in this work with other estimations also photometrically obtained. The equality line is plotted in all panels as a dashed line. The photometric effective temperatures agree with those spectroscopically derived. That is not the case of the radii, where intrinsic errors when applying flux calibration induce a systematic error in the radius calculation in the spectroscopic method, hence in the bolometric luminosities also. On the contrary, the luminosities derived by our photometric procedure are in perfect agreement with those published in the literature (see text for details).

derived spectroscopically. That is a consequence of systematically lower spectroscopic radii, as previously stated. For this reason, we do not consider the spectroscopic radii (hence bolometric luminosities) to be as reliable as the photometric ones and hence decided not to make use of these values.

Finally, we carry out an additional verification comparing the photometric luminosity of our work with other values published in the literature and also derived photometrically (Kilic et al. 2018; Tremblay et al. 2020). The results are shown in the bottom right panel of Fig. 4. As it can be seen, the agreement is excellent. Thus, our photometric procedure provides effective temperatures which are in agreement with those derived spectroscopically and yields luminosi- ties which are in perfect agreement with those also derived photometrically in the literature. Consequently, we can conclude that the rest of parameters are robustly obtained and derived in a consistent way by our photometric method.

Once we have demonstrated that our strategy is feasible to compute the white dwarf luminosities we extend it to the whole halo white dwarf sample. Analogously, we also derive the rest of parameters: mass, age, effective temperature and radius. The only exception is for object J0055+3847, which is located, as previously stated, outside the region of the HR-diagram covered by the single white dwarf population (see Fig. 1). In Table 2 we present the parameters thus derived from our photometric method. Along with the Gaia source ID (first 


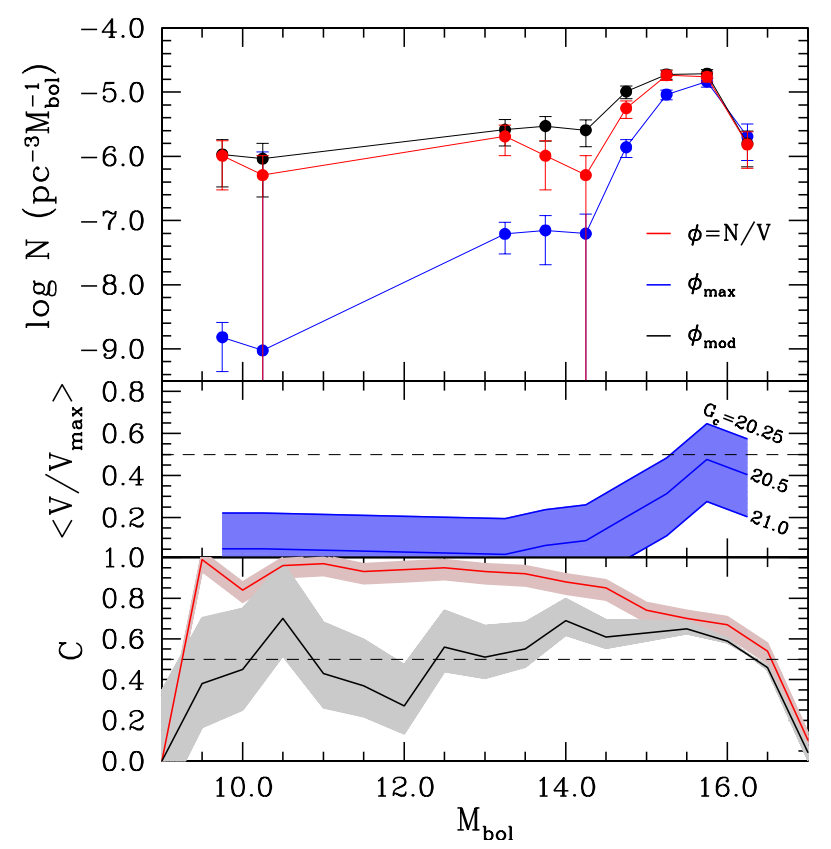

Figure 5. Top panel: Halo white dwarf luminosity functions built with the classical estimator $\phi=N / V$ (red line and dots), with the $1 / \mathcal{V}_{\max }$ method, $\phi_{\max }$ (blue line and dots), and with the modified volume, $\phi_{\text {mod }}$ (black line and dots). Middle panel: average $\left\langle V / V_{\max }\right\rangle$ as a function of the bolometric magnitude for different magnitude cuts, $G_{c} \in[20.25,21.0]$ mag. Also shown as a dashed line the value representing an uniform complete sample, $\left\langle V / V_{\max }\right\rangle=0.5$. Bottom panel: completeness of the whole Gaia $100 \mathrm{pc}$ sample of white dwarfs (red line, $1 \sigma$ pink area) and that of the halo subsample (black line, $1 \sigma$ gray area).

column) and the short name (second column), we show the absolute magnitude and colour and their respective errors used in building our ellipsoids (columns third to sixth) and the mass, effective temperature, bolometric luminosity and age, with their respective errors (columns seventh to tenth). It is worth recalling that our photometric method assumes a mixed population of $80 \%$ DA and $20 \%$ DB white dwarfs, and then just counts the physical parameters not caring what the atmospheric composition is.

\subsection{Completeness analysis of the halo white dwarf sample}

As a preliminary step for building the halo white dwarf luminosity function, we analyze in detail the completeness of our selected sample. First, we need to take into account that our 'halo sample' is not a 'direct' observed sample. That is, we first obtain a sample of white dwarfs within $100 \mathrm{pc}$, and from that we apply a classifying method based on a Random Forest algorithm in order to obtain the 'halo sample'. Then, the incompleteness raises from two sources: from the selection process of the observed 100 pc sample and from the classification algorithm.

With respect to the first item, the Gaia mission is expected to be (understood as an end-of-mission goal) complete up to $G=21$ mag. Recent estimates based on the EDR3-Gaia catalogue of nearby stars provide a completeness of $97 \%, 95 \%$ and $91 \%$ for $G$ magnitudes 19.9, 20.2 and 20.5, respectively (Gaia Collaboration et al. 2020). Taking this into account, we extend our completeness analysis of the $100 \mathrm{pc}$ sample (see Figure 5 from Jiménez-Esteban et al. (2018)) up to $G_{B P}-G_{R P}=2.0$. The resulting completeness as a function of the bolometric magnitude for the whole Gaia 100 pc sample is shown in the bottom panel (red line) of Figure 5. A completeness above $90 \%$ is achieved for most of the sample up to $M_{\text {bol }} \sim 14.0$. At this magnitude, we note a decreasing trend reaching a 50\% completeness at magnitude $M_{\text {bol }} \sim 16.6$ mag.

Secondly, we analyze the incompleteness introduced by our Random Forest classification algorithm. We recall that in our classification process we used an 8-dimensional space (equatorial coordinates, parallax, proper motion components and photometric magnitudes), where the algorithm estimates the entropy function, evaluated on each splitting branch of the different decisions trees of the Random Forest algorithm (see Torres et al. 2019a). For each individual object the minimum entropy found, $S_{i}$, is used to classify the object in a certain Galactic component. Consequently, this entropy value $S_{i}$ can be logically understood as an inverse of the probability that an object belongs to a certain group. Using our Monte Carlo simulator we generate several samples of $\sim 95$ halo white dwarfs and calculate, through our Random Forest algorithm, the individual probability of being classified as such. We compute, then, the average value as a function of the bolometric magnitude taking into account the completeness of the whole $100 \mathrm{pc}$ sample. The resulting distribution is shown in the bottom panel of Figure 5 as a black line (gray area corresponding to $1 \sigma$ dispersion). We observe an irregular pattern for the bright region $\left(M_{\text {bol }} \lesssim 14\right.$ mag), while for fainter magnitudes the halo white dwarf completeness distribution resembles that of the whole sample. According to our analysis, the noisy appearance of the bright region is due to two factors: mainly, the low number of objects expected in these bins, which are dominated by Poisson counting error statistics, and secondly, and to a minor extent, to the intrinsic difficulty to classify a bright object as belonging to the halo population. High-speed and cool objects are closer to what we expect to be a halo white dwarf than hot and high-speed objects. In the first case, the automatic classification algorithm seems to relax the kinematic condition to accept moderately fast objects if they are cool enough, while the reverse condition happens for hot halo candidates. Besides, more hot high-speed disk objects may contaminate the hot region and the difficulty to disentangle halo objects increases in that region. On the other hand, for fainter magnitudes the halo sample completeness resembles that of the whole $100 \mathrm{pc}$ sample, indicating that the probability to correctly classifying a halo object in this region is very high. It should be noted that the completeness of the halo sample for magnitudes around $M_{\text {bol }}=16.0$ is $\sim 60 \%$, which can be considered as an acceptable value, while for the rest of the sample is around $\sim 50 \%$.

We are in position now to estimate the luminosity function. Among the different estimators proposed (see, for instance, Geijo et al. 2006 and reference therein) the $1 / \mathcal{V}_{\max }$ method (Schmidt 1968; Felten 1976 ) is the most commonly used to estimate the white dwarf luminosity function. However, this method, based on the assumption of a complete magnitude-limited sample, is not applicable in our case, since our classification method introduces an incompleteness factor which is not only magnitude dependent. Consequently, we will use the $1 / \mathcal{V}_{\max }$ method for comparative purposes just as a first guess in our analysis. In the middle panel of Figure 5 we show the average $\left\langle V / V_{\max }\right\rangle$ as function of $M_{\text {bol }}$ adopting different $G$ magnitude cuts, $G_{c}$, of the whole $100 \mathrm{pc}$ sample in the range $G_{c} \in[20.25,21.0]$ mags. We also recall that the $1 / \mathcal{V}_{\max }$ provides an estimation of the completeness of the sample, once assumed a complete uniformly distributed sample, resulting in a average value of $\left\langle V / V_{\max }\right\rangle=0.5$. In our case, adopting a conservative value of $G_{c}=20.5$ mags, the $\left\langle V / V_{\max }\right\rangle$ distribution is closer to the value 0.5 (dashed line) for magnitudes $M_{\mathrm{bol}} \sim 15.7$ mags. Specifically, a value 
Table 2. Stellar parameters derived by our photometric method for the whole halo white dwarf sample within 100 pc. Along with the Gaia Source ID and short name (first and second columns, respectively), the location within the HR-diagram (third and fourth columns) and the size of the adopted ellipses (fifth and six columns) are shown. Radius, effective temperature, bolometric luminosity and total age are shown, respectively, in the last four columns.

\begin{tabular}{|c|c|c|c|c|c|c|c|c|c|}
\hline $\begin{array}{c}\text { Gaia } \\
\text { Source ID }\end{array}$ & $\begin{array}{l}\text { Short } \\
\text { name }\end{array}$ & $\begin{array}{c}M_{\mathrm{G}} \\
(\mathrm{mag})\end{array}$ & $\begin{array}{c}G_{\mathrm{BP}}-G_{\mathrm{RP}} \\
\quad(\mathrm{mag})\end{array}$ & $\begin{array}{l}\sigma_{M_{\mathrm{G}}} \\
(\mathrm{mag})\end{array}$ & $\begin{array}{c}\sigma_{G_{\mathrm{BP}}-G_{\mathrm{RP}}} \\
(\mathrm{mag})\end{array}$ & $\begin{array}{c}\text { Mass } \\
M_{\odot}\end{array}$ & $\begin{array}{l}T_{\text {eff }} \\
(\mathrm{K})\end{array}$ & $\begin{array}{l}\text { Luminosity } \\
\log \left(L / L_{\odot}\right)\end{array}$ & $\begin{array}{l}\text { Age } \\
(\mathrm{Gyr})\end{array}$ \\
\hline 420531621029108608 & $\mathrm{~J} 0013+5438$ & 15.47 & 1.47 & 0.007 & 0.041 & $0.551 \pm 0.027$ & $4306 \pm 73$ & $-4.273 \pm 0.007$ & $11.27 \pm 1.83$ \\
\hline 5006232026455470848 & J0045-3329 & 16.05 & 1.51 & 0.011 & 0.072 & $0.613 \pm 0.051$ & $3829 \pm 128$ & $-4.544 \pm 0.019$ & $12.22 \pm 1.48$ \\
\hline 367799116372410752 & $\mathrm{~J} 0055+3847$ & 13.51 & 0.89 & 0.015 & 0.037 & & $\ldots$ & $\ldots$ & $\ldots$ \\
\hline 2583365245917474816 & $\mathrm{~J} 0106+1141$ & 15.76 & 1.43 & 0.039 & 0.169 & $0.599 \pm 0.065$ & $4112 \pm 163$ & $-4.404 \pm 0.013$ & $11.43 \pm 1.39$ \\
\hline 5042228731477861888 & J0129-2257 & 15.54 & 1.41 & 0.086 & 0.282 & $0.589 \pm 0.067$ & $4333 \pm 170$ & $-4.302 \pm 0.022$ & $10.56 \pm 1.56$ \\
\hline 96095735719745280 & $\mathrm{~J} 0132+1941$ & 14.85 & 1.13 & 0.049 & 0.145 & $0.556 \pm 0.049$ & $4941 \pm 130$ & $-4.033 \pm 0.015$ & $8.36 \pm 2.41$ \\
\hline 5142197118950177280 & J0148-1712 & 13.11 & 0.46 & 0.011 & 0.025 & $0.529 \pm 0.023$ & $7286 \pm 124$ & $-3.311 \pm 0.010$ & $6.52 \pm 2.66$ \\
\hline 343356212681211392 & $\mathrm{~J} 0157+3932$ & 15.64 & 1.44 & 0.046 & 0.187 & $0.585 \pm 0.054$ & $4205 \pm 135$ & $-4.350 \pm 0.013$ & $11.03 \pm 1.44$ \\
\hline 521409549427243904 & J0158+6904 & 15.47 & 1.14 & 0.103 & 0.298 & $0.630 \pm 0.108$ & $4516 \pm 279$ & $-4.272 \pm 0.024$ & $9.80 \pm 1.60$ \\
\hline 2490975272405858048 & J0205-0517 & 15.58 & 1.52 & 0.008 & 0.046 & $0.544 \pm 0.019$ & $4165 \pm 57$ & $-4.325 \pm 0.009$ & $11.81 \pm 1.42$ \\
\hline 4616895783694397184 & J0237-8445 & 15.74 & 1.55 & 0.039 & 0.176 & $0.579 \pm 0.047$ & $4086 \pm 117$ & $-4.394 \pm 0.012$ & $11.62 \pm 1.38$ \\
\hline 5065611697758431360 & J0248-3001 & 13.91 & 0.71 & 0.019 & 0.050 & $0.573 \pm 0.040$ & $6143 \pm 162$ & $-3.669 \pm 0.011$ & $4.85 \pm 2.02$ \\
\hline 5188044687948351872 & J0301-0044 & 15.24 & 1.51 & 0.041 & 0.157 & $0.543 \pm 0.033$ & $4516 \pm 86$ & $-4.181 \pm 0.012$ & $10.60 \pm 1.79$ \\
\hline 4862884499360563968 & J0340-3301 & 15.79 & 1.31 & 0.032 & 0.143 & $0.636 \pm 0.087$ & $4177 \pm 220$ & $-4.414 \pm 0.012$ & $11.17 \pm 1.23$ \\
\hline 3249657094642979840 & J0342-0344 & 15.46 & 1.09 & 0.057 & 0.194 & $0.680 \pm 0.112$ & $4646 \pm 285$ & $-4.272 \pm 0.016$ & $9.21 \pm 1.28$ \\
\hline 4857106909354185344 & J0345-3611 & 15.37 & 1.47 & 0.061 & 0.212 & $0.559 \pm 0.043$ & $4422 \pm 110$ & $-4.235 \pm 0.015$ & $10.54 \pm 1.74$ \\
\hline 66837563803594880 & $\mathrm{~J} 0346+2455$ & 15.58 & 1.50 & 0.013 & 0.069 & $0.553 \pm 0.031$ & $4197 \pm 83$ & $-4.322 \pm 0.009$ & $11.49 \pm 1.51$ \\
\hline 4864861112027378944 & J0431-3816 & 15.75 & 1.77 & 0.133 & 0.456 & $0.584 \pm 0.053$ & $4089 \pm 143$ & $-4.399 \pm 0.029$ & $11.54 \pm 1.37$ \\
\hline 4864752883148064512 & J0432-3902 & 14.86 & 1.25 & 0.005 & 0.025 & $0.527 \pm 0.021$ & $4846 \pm 57$ & $-4.037 \pm 0.013$ & $9.86 \pm 2.08$ \\
\hline 2989049057626796416 & J0518-1155 & 15.70 & 1.74 & 0.079 & 0.306 & $0.568 \pm 0.040$ & $4102 \pm 104$ & $-4.377 \pm 0.017$ & $11.69 \pm 1.32$ \\
\hline 192454873200555392 & $\mathrm{~J} 0559+4248$ & 13.42 & 0.52 & 0.022 & 0.049 & $0.578 \pm 0.035$ & $6992 \pm 172$ & $-3.445 \pm 0.010$ & $3.71 \pm 1.60$ \\
\hline 977441274176008192 & $\mathrm{~J} 0711+4607$ & 14.76 & 1.16 & 0.033 & 0.105 & $0.536 \pm 0.034$ & $4983 \pm 93$ & $-3.996 \pm 0.014$ & $9.03 \pm 2.42$ \\
\hline 5613373001468333696 & J0732-2558 & 15.11 & 1.27 & 0.097 & 0.265 & $0.568 \pm 0.059$ & $4714 \pm 155$ & $-4.130 \pm 0.023$ & $9.20 \pm 2.14$ \\
\hline 874900643675606912 & $\mathrm{~J} 0745+2626$ & 15.76 & 1.60 & 0.014 & 0.081 & $0.572 \pm 0.046$ & $4046 \pm 112$ & $-4.406 \pm 0.012$ & $11.80 \pm 1.40$ \\
\hline 1110759459929880704 & J0748+7141 & 15.74 & 1.62 & 0.062 & 0.251 & $0.579 \pm 0.048$ & $4078 \pm 124$ & $-4.398 \pm 0.016$ & $11.67 \pm 1.37$ \\
\hline 3144837318276010624 & $\mathrm{~J} 0750+0711 \mathrm{a}$ & 15.07 & 1.25 & 0.002 & 0.013 & $0.530 \pm 0.023$ & $4664 \pm 56$ & $-4.108 \pm 0.006$ & $9.54 \pm 2.07$ \\
\hline 3144837112117580800 & $\mathrm{~J} 0750+0711 \mathrm{~b}$ & 15.31 & 1.41 & 0.002 & 0.010 & $0.565 \pm 0.025$ & $4512 \pm 59$ & $-4.202 \pm 0.006$ & $10.79 \pm 1.19$ \\
\hline 5726927573083821440 & J0822-1249 & 15.54 & 1.30 & 0.037 & 0.148 & $0.597 \pm 0.069$ & $4346 \pm 170$ & $-4.305 \pm 0.012$ & $10.40 \pm 1.48$ \\
\hline 5742629217603133056 & J0912-0953 & 15.27 & 1.15 & 0.094 & 0.265 & $0.606 \pm 0.090$ & $4656 \pm 230$ & $-4.192 \pm 0.023$ & $9.17 \pm 1.86$ \\
\hline 5215833263797633664 & J0913-7553 & 15.37 & 1.37 & 0.038 & 0.147 & $0.562 \pm 0.046$ & $4439 \pm 116$ & $-4.231 \pm 0.013$ & $10.39 \pm 1.80$ \\
\hline 3840846114438361984 & $\mathrm{~J} 0925+0018$ & 15.12 & 1.15 & 0.032 & 0.115 & $0.578 \pm 0.061$ & $4729 \pm 155$ & $-4.134 \pm 0.013$ & $8.83 \pm 2.02$ \\
\hline 1064978578888570496 & $\mathrm{~J} 0941+6511$ & 15.14 & 1.34 & 0.013 & 0.059 & $0.539 \pm 0.032$ & $4610 \pm 83$ & $-4.139 \pm 0.011$ & $10.49 \pm 2.04$ \\
\hline 3836593100382315904 & $\mathrm{~J} 1005+0254$ & 15.26 & 1.29 & 0.065 & 0.207 & $0.574 \pm 0.058$ & $4580 \pm 148$ & $-4.187 \pm 0.018$ & $9.62 \pm 1.91$ \\
\hline 746045096445123968 & $\mathrm{~J} 1012+3233$ & 15.70 & 1.39 & 0.131 & 0.399 & $0.615 \pm 0.091$ & $4224 \pm 240$ & $-4.374 \pm 0.034$ & $11.02 \pm 1.45$ \\
\hline 5192296911732427904 & J1036-8225 & 15.67 & 1.54 & 0.040 & 0.176 & $0.573 \pm 0.044$ & $4150 \pm 109$ & $-4.361 \pm 0.011$ & $11.37 \pm 1.37$ \\
\hline 3862858165427681536 & $\mathrm{~J} 1036+0732$ & 15.22 & 1.49 & 0.023 & 0.098 & $0.540 \pm 0.027$ & $4530 \pm 75$ & $-4.171 \pm 0.010$ & $10.63 \pm 1.69$ \\
\hline 1076941716370493696 & $\mathrm{~J} 1036+7110$ & 15.39 & 1.24 & 0.002 & 0.012 & $0.582 \pm 0.071$ & $4461 \pm 165$ & $-4.241 \pm 0.009$ & $10.52 \pm 1.65$ \\
\hline 855361055035055104 & $\mathrm{~J} 1045+5904$ & 13.89 & 0.23 & 0.009 & 0.029 & $1.074 \pm 0.033$ & $9211 \pm 481$ & $-3.527 \pm 0.024$ & $2.69 \pm 0.25$ \\
\hline 5228861484450843648 & J1049-7400 & 16.07 & 1.54 & 0.021 & 0.122 & $0.613 \pm 0.054$ & $3813 \pm 134$ & $-4.551 \pm 0.020$ & $12.23 \pm 1.54$ \\
\hline 3801499128765222400 & J1053-0307 & 15.37 & 1.42 & 0.041 & 0.156 & $0.556 \pm 0.042$ & $4422 \pm 107$ & $-4.232 \pm 0.013$ & $10.62 \pm 1.77$ \\
\hline 3865951435233552896 & $\mathrm{~J} 1055+0816$ & 15.46 & 1.67 & 0.043 & 0.181 & $0.556 \pm 0.042$ & $4324 \pm 108$ & $-4.271 \pm 0.014$ & $11.14 \pm 1.62$ \\
\hline 1055533400343235456 & $\mathrm{~J} 1101+6333$ & 15.33 & 1.44 & 0.068 & 0.226 & $0.560 \pm 0.045$ & $4464 \pm 115$ & $-4.219 \pm 0.017$ & $10.31 \pm 1.81$ \\
\hline 831946229073235200 & $\mathrm{~J} 1107+4855$ & 15.19 & 1.27 & 0.015 & 0.066 & $0.552 \pm 0.041$ & $4588 \pm 104$ & $-4.162 \pm 0.011$ & $9.97 \pm 1.94$ \\
\hline 5348874243767794304 & $\mathrm{~J} 1123-5150$ & 15.58 & 1.32 & 0.069 & 0.242 & $0.604 \pm 0.080$ & $4325 \pm 204$ & $-4.321 \pm 0.018$ & $10.57 \pm 1.47$ \\
\hline 856513235846126720 & $\mathrm{~J} 1123+5742$ & 14.74 & 0.65 & 0.061 & 0.159 & $0.893 \pm 0.100$ & $6163 \pm 418$ & $-3.997 \pm 0.017$ & $5.15 \pm 0.24$ \\
\hline 5224999346778496128 & J1147-7457 & 15.66 & 1.58 & 0.003 & 0.021 & $0.575 \pm 0.037$ & $4159 \pm 95$ & $-4.357 \pm 0.005$ & $11.58 \pm 1.20$ \\
\hline 3892524535332945280 & $\mathrm{~J} 1151+0159$ & 15.13 & 1.24 & 0.061 & 0.188 & $0.564 \pm 0.054$ & $4683 \pm 139$ & $-4.138 \pm 0.017$ & $9.35 \pm 2.11$ \\
\hline 5377861317357370240 & J1159-4630 & 15.17 & 1.24 & 0.029 & 0.109 & $0.561 \pm 0.046$ & $4630 \pm 118$ & $-4.154 \pm 0.013$ & $9.54 \pm 2.00$ \\
\hline 5377861592235273856 & J1159-4629 & 13.44 & 0.41 & 0.009 & 0.023 & $0.705 \pm 0.051$ & $7589 \pm 241$ & $-3.437 \pm 0.016$ & $2.32 \pm 0.37$ \\
\hline 1573358945589364608 & $\mathrm{~J} 1205+5502$ & 15.84 & 1.42 & 0.106 & 0.360 & $0.623 \pm 0.089$ & $4091 \pm 233$ & $-4.439 \pm 0.030$ & $11.50 \pm 1.39$ \\
\hline 3905186270720273152 & $\mathrm{~J} 1217+0830$ & 15.72 & 1.31 & 0.075 & 0.264 & $0.619 \pm 0.089$ & $4219 \pm 227$ & $-4.380 \pm 0.019$ & $10.99 \pm 1.43$ \\
\hline 1533950318546008448 & $\mathrm{~J} 1235+4109$ & 15.47 & 1.59 & 0.041 & 0.193 & $0.563 \pm 0.053$ & $4333 \pm 136$ & $-4.272 \pm 0.008$ & $10.66 \pm 1.21$ \\
\hline 1570514066627694336 & $\mathrm{~J} 1250+5446$ & 18.01 & 1.75 & 0.004 & 0.024 & $0.563 \pm 0.038$ & $4033 \pm 97$ & $-4.402 \pm 0.014$ & $11.93 \pm 1.25$ \\
\hline 1531097433767946240 & $\mathrm{~J} 1255+4655$ & 15.57 & 1.17 & 0.011 & 0.055 & $0.728 \pm 0.112$ & $4641 \pm 286$ & $-4.322 \pm 0.010$ & $9.52 \pm 0.99$ \\
\hline 1459546263999675264 & $\mathrm{~J} 1303+2603$ & 15.51 & 1.45 & 0.011 & 0.058 & $0.562 \pm 0.041$ & $4293 \pm 106$ & $-4.290 \pm 0.009$ & $11.06 \pm 1.56$ \\
\hline 6085402414245451520 & J1312-4728 & 15.58 & 1.53 & 0.002 & 0.014 & $0.542 \pm 0.002$ & $4126 \pm 19$ & $-4.337 \pm 0.006$ & $12.41 \pm 0.22$ \\
\hline 3607725941130742528 & J1316-1536 & 11.09 & -0.20 & 0.002 & 0.004 & $0.551 \pm 0.015$ & $15778 \pm 71$ & $-1.991 \pm 0.015$ & $2.37 \pm 0.60$ \\
\hline 6188655210447329792 & J1338-2747 & 15.68 & 1.50 & 0.085 & 0.302 & $0.594 \pm 0.064$ & $4190 \pm 162$ & $-4.367 \pm 0.021$ & $11.09 \pm 1.46$ \\
\hline 6165095738576250624 & $\mathrm{~J} 1342-3415$ & 14.66 & 1.05 & 0.002 & 0.009 & $0.521 \pm 0.013$ & $5055 \pm 39$ & $-3.956 \pm 0.010$ & $9.45 \pm 1.86$ \\
\hline 3714266139665215488 & $\mathrm{~J} 1348+0527$ & 15.03 & 1.20 & 0.086 & 0.233 & $0.566 \pm 0.058$ & $4790 \pm 153$ & $-4.099 \pm 0.022$ & $8.89 \pm 2.25$ \\
\hline
\end{tabular}

MNRAS 000, 1-15 (2015) 
Table 2 - continued

\begin{tabular}{|c|c|c|c|c|c|c|c|c|c|}
\hline $\begin{array}{c}\text { Gaia } \\
\text { Source ID }\end{array}$ & $\begin{array}{l}\text { Short } \\
\text { name }\end{array}$ & $\begin{array}{c}M_{\mathrm{G}} \\
\text { (mag) }\end{array}$ & $\begin{array}{c}G_{\mathrm{BP}}-G_{\mathrm{RP}} \\
\quad(\mathrm{mag})\end{array}$ & $\begin{array}{l}\sigma_{M_{\mathrm{G}}} \\
(\mathrm{mag})\end{array}$ & $\begin{array}{c}\sigma_{G_{\mathrm{BP}}-G_{\mathrm{RP}}} \\
(\mathrm{mag})\end{array}$ & $\begin{array}{c}\text { Mass } \\
M_{\odot}\end{array}$ & $\begin{array}{l}T_{\text {eff }} \\
(\mathrm{K})\end{array}$ & $\begin{array}{l}\text { Luminosity } \\
\log \left(L / L_{\odot}\right)\end{array}$ & $\begin{array}{l}\text { Age } \\
(\mathrm{Gyr})\end{array}$ \\
\hline 1174809276422844160 & $\mathrm{~J} 1442+1003$ & 15.51 & 1.40 & 0.105 & 0.324 & $0.593 \pm 0.071$ & $4366 \pm 183$ & $-4.292 \pm 0.026$ & $10.43 \pm 1.58$ \\
\hline 1161215296909017728 & $\mathrm{~J} 1450+0733$ & 11.14 & -0.17 & 0.004 & 0.006 & $0.537 \pm 0.015$ & $14638 \pm 180$ & $-2.069 \pm 0.015$ & $3.46 \pm 1.36$ \\
\hline 1294793345366747776 & $\mathrm{~J} 1500+3600$ & 14.65 & 1.17 & 0.034 & 0.102 & $0.530 \pm 0.031$ & $5077 \pm 89$ & $-3.956 \pm 0.013$ & $9.19 \pm 2.50$ \\
\hline 1600259390916467072 & $\mathrm{~J} 1502+5409$ & 15.33 & 1.42 & 0.060 & 0.204 & $0.559 \pm 0.044$ & $4466 \pm 113$ & $-4.217 \pm 0.016$ & $10.33 \pm 1.82$ \\
\hline 1612339420228653440 & $\mathrm{~J} 1503+5509$ & 15.76 & 1.36 & 0.073 & 0.265 & $0.615 \pm 0.083$ & $4163 \pm 210$ & $-4.399 \pm 0.020$ & $11.23 \pm 1.38$ \\
\hline 5824436284328653312 & $\mathrm{~J} 1517-6645$ & 15.99 & 1.30 & 0.102 & 0.359 & $0.661 \pm 0.114$ & $4029 \pm 307$ & $-4.506 \pm 0.029$ & $11.70 \pm 1.56$ \\
\hline 6007140379167609984 & $\mathrm{~J} 1518-3803$ & 15.66 & 1.55 & 0.040 & 0.173 & $0.571 \pm 0.043$ & $4151 \pm 108$ & $-4.359 \pm 0.011$ & $11.39 \pm 1.36$ \\
\hline 1277219369981634432 & $\mathrm{~J} 1522+3146$ & 15.70 & 1.54 & 0.103 & 0.351 & $0.596 \pm 0.066$ & $4171 \pm 170$ & $-4.376 \pm 0.025$ & $11.18 \pm 1.45$ \\
\hline 1277232907719022464 & $\mathrm{~J} 1523+3152$ & 15.59 & 1.26 & 0.021 & 0.098 & $0.626 \pm 0.079$ & $4364 \pm 197$ & $-4.326 \pm 0.010$ & $10.30 \pm 1.35$ \\
\hline 5827557213731539328 & J1539-6124 & 15.18 & 1.31 & 0.026 & 0.104 & $0.550 \pm 0.039$ & $4593 \pm 100$ & $-4.157 \pm 0.012$ & $10.05 \pm 1.97$ \\
\hline 5817295536128445568 & J1707-6319 & 15.10 & 1.40 & 0.019 & 0.079 & $0.533 \pm 0.029$ & $4637 \pm 74$ & $-4.123 \pm 0.012$ & $10.68 \pm 2.00$ \\
\hline 5802598780807649920 & $\mathrm{~J} 1715-7323$ & 15.43 & 1.09 & 0.111 & 0.310 & $0.640 \pm 0.116$ & $4586 \pm 304$ & $-4.255 \pm 0.025$ & $9.50 \pm 1.63$ \\
\hline 1711005951573009792 & $\mathrm{~J} 1749+8247$ & 13.15 & 0.47 & 0.001 & 0.003 & $0.547 \pm 0.000$ & $7257 \pm 0$ & $-3.331 \pm 0.000$ & $4.33 \pm 0.00$ \\
\hline 6363668569344689408 & $\mathrm{~J} 1812-8028$ & 15.55 & 1.04 & 0.143 & 0.390 & $0.659 \pm 0.131$ & $4520 \pm 353$ & $-4.302 \pm 0.029$ & $9.85 \pm 1.54$ \\
\hline 6653858618815379328 & $\mathrm{~J} 1814-5305$ & 15.68 & 1.08 & 0.130 & 0.376 & $0.669 \pm 0.132$ & $4396 \pm 356$ & $-4.361 \pm 0.027$ & $10.41 \pm 1.47$ \\
\hline 4484289866726156160 & $\mathrm{~J} 1824+1213$ & 15.57 & 1.63 & 0.012 & 0.069 & $0.548 \pm 0.025$ & $4195 \pm 74$ & $-4.316 \pm 0.011$ & $11.72 \pm 1.25$ \\
\hline 2146619161278293248 & $\mathrm{~J} 1852+5333$ & 15.72 & 1.39 & 0.134 & 0.410 & $0.617 \pm 0.093$ & $4216 \pm 245$ & $-4.379 \pm 0.035$ & $11.06 \pm 1.44$ \\
\hline 6663268308043562112 & J1926-4627 & 15.02 & 1.46 & 0.060 & 0.190 & $0.533 \pm 0.029$ & $4711 \pm 79$ & $-4.095 \pm 0.016$ & $10.25 \pm 2.06$ \\
\hline 6647162730439433984 & J1936-4913 & 15.58 & 1.50 & 0.057 & 0.219 & $0.574 \pm 0.047$ & $4251 \pm 121$ & $-4.320 \pm 0.015$ & $10.94 \pm 1.47$ \\
\hline 2301882675705225472 & $\mathrm{~J} 1940+8348$ & 15.51 & 1.23 & 0.010 & 0.049 & $0.608 \pm 0.065$ & $4413 \pm 155$ & $-4.290 \pm 0.012$ & $9.78 \pm 1.39$ \\
\hline 2082254987541672960 & $\mathrm{~J} 2006+4544$ & 15.21 & 1.22 & 0.037 & 0.136 & $0.574 \pm 0.057$ & $4629 \pm 143$ & $-4.169 \pm 0.013$ & $9.32 \pm 1.93$ \\
\hline 6471523921227261056 & $\mathrm{~J} 2042-5218$ & 14.68 & 0.94 & 0.043 & 0.121 & $0.601 \pm 0.070$ & $5249 \pm 199$ & $-3.973 \pm 0.015$ & $6.22 \pm 1.96$ \\
\hline 1737588947276271744 & $\mathrm{~J} 2052+0709$ & 15.89 & 1.56 & 0.059 & 0.250 & $0.605 \pm 0.061$ & $3980 \pm 158$ & $-4.468 \pm 0.020$ & $11.78 \pm 1.38$ \\
\hline 6580458035746362496 & $\mathrm{~J} 2117-4156 \mathrm{a}$ & 15.15 & 1.33 & 0.024 & 0.095 & $0.542 \pm 0.033$ & $4608 \pm 84$ & $-4.143 \pm 0.012$ & $10.28 \pm 1.96$ \\
\hline 6580551872194787968 & $\mathrm{~J} 2117-4156 \mathrm{~b}$ & 15.34 & 1.30 & 0.028 & 0.115 & $0.572 \pm 0.052$ & $4493 \pm 130$ & $-4.220 \pm 0.013$ & $9.94 \pm 1.78$ \\
\hline 1783614400935169408 & $\mathrm{~J} 2127+1545$ & 14.30 & 0.82 & 0.023 & 0.066 & $0.599 \pm 0.058$ & $5700 \pm 191$ & $-3.827 \pm 0.012$ & $5.06 \pm 1.86$ \\
\hline 2687584757658775424 & J2129-0034 & 15.37 & 1.57 & 0.112 & 0.341 & $0.562 \pm 0.045$ & $4427 \pm 120$ & $-4.236 \pm 0.022$ & $10.46 \pm 1.76$ \\
\hline 6465689878168451328 & $\mathrm{~J} 2139-5058$ & 15.85 & 1.55 & 0.084 & 0.320 & $0.607 \pm 0.067$ & $4025 \pm 175$ & $-4.450 \pm 0.025$ & $11.68 \pm 1.33$ \\
\hline 2205493129867600256 & $\mathrm{~J} 2225+6357$ & 14.53 & 1.05 & 0.006 & 0.024 & $0.525 \pm 0.020$ & $5190 \pm 63$ & $-3.912 \pm 0.012$ & $8.98 \pm 2.20$ \\
\hline 6357629089412187648 & $\mathrm{~J} 2230-7515$ & 15.57 & 1.55 & 0.002 & 0.012 & $0.575 \pm 0.059$ & $4275 \pm 157$ & $-4.313 \pm 0.011$ & $10.61 \pm 0.99$ \\
\hline 2709539840202060800 & $\mathrm{~J} 2237+0636$ & 15.85 & 1.54 & 0.110 & 0.396 & $0.617 \pm 0.081$ & $4058 \pm 211$ & $-4.446 \pm 0.031$ & $11.60 \pm 1.37$ \\
\hline 1941133391670459648 & $\mathrm{~J} 2314+4545$ & 15.96 & 1.41 & 0.099 & 0.358 & $0.640 \pm 0.095$ & $4008 \pm 248$ & $-4.492 \pm 0.029$ & $11.74 \pm 1.44$ \\
\hline 2631967439437024384 & J2319-0613 & 15.06 & 1.37 & 0.007 & 0.035 & $0.530 \pm 0.023$ & $4661 \pm 52$ & $-4.112 \pm 0.013$ & $10.40 \pm 1.68$ \\
\hline 2641576685735609472 & J2349-0124 & 14.99 & 1.22 & 0.028 & 0.099 & $0.544 \pm 0.038$ & $4771 \pm 97$ & $-4.083 \pm 0.013$ & $9.43 \pm 2.17$ \\
\hline 2310942857676734848 & $\mathrm{~J} 2354-3634$ & 11.29 & -0.16 & 0.003 & 0.005 & $0.574 \pm 0.018$ & $14636 \pm 121$ & $-2.134 \pm 0.005$ & $1.68 \pm 0.49$ \\
\hline
\end{tabular}

of $\left\langle V / V_{\max }\right\rangle=0.476 \pm 0.049$ (adopting for $N$ objects a deviation of $1 / \sqrt{12 N}$, Rowell \& Hambly 2011) thus indicating an acceptable degree of completeness for that particular magnitude bin. However, as we mentioned earlier, brighter magnitudes appear to be far from being complete as shown by the low $\left\langle V / V_{\max }\right\rangle$ value.

In the top panel of Figure 5, we compare the different halo white dwarfs luminosity functions built from our previous completeness analysis. First, we use the classical estimator of the luminosity function for volume-limited samples, $\phi=N / V$. The resulting luminosity function is shown in red. Second, the $1 / \mathcal{V}_{\max }$ provides an estimation of the luminosity function, $\phi_{\max }$, binning the sample in $i \in(1, N)$ magnitude bins and weighting the contribution of each object as inversely proportional to its maximum volume available within the selection cuts: $\phi_{\max }=\sum_{j=1}^{N_{i}} 1 / \mathcal{V}_{\max }^{j}$, where the deviation is calculated as the sum in quadrature of the individual errors. Adopting a magnitude cut of $G_{C}=20.5 \mathrm{mag}$, the luminosity function thus obtained is shown in blue in the top panel of Fig. 5. However, as previously stated, both, classical and $1 / \mathcal{V}_{\max }$ method, can be only understood as a first guess for building a proper luminosity function.

In order to properly take into account all possible sources of incompleteness in our sample, as previously discussed, we use a gen- eralization of the $1 / \mathcal{V}_{\max }$ method (Lam et al. 2015 and references therein). A modified volume is defined as

$\mathcal{V}_{\text {mod }}^{i}=\Omega_{i} \int_{r_{\text {min }}}^{r_{\text {max }}} \chi \frac{\rho(r)}{\rho_{\odot}} r^{2} d r$

where $\Omega_{i}$ is the solid angle covered by object $i, r_{\max }$ and $r_{\min }$ are, respectively the maximum and minimum distances and $\rho(r) / \rho_{\odot}$ the density ratio along the line of side. This volume integral is modified by the function $\chi$, traditionally named as the discovery fraction, and typically used for taking into account the number of objects that pass a certain tangential velocity threshold. In our case, we generalize the $\chi$ function to incorporate our 8-dimensional space of equatorial coordinates, proper motion components, parallax and photometric magnitudes, $\chi=\chi\left(\alpha, \delta, \mu_{\alpha}^{*}, \mu_{\delta}, \varpi, G, G_{R P}, G_{B P}\right)$. Thus, the $\chi$ function becomes equivalent to the probability derived from our Random Forest algorithm of being correctly classified as an halo member. Adopting a constant density profile, which is a natural assumption for a halo distribution in a small volume, we can compute the luminosity function as a generalization of the $1 / \mathcal{V}_{\max }$ method by using the previously modified volume: $\phi_{\bmod }=\sum_{j=1}^{N_{i}} 1 / \mathcal{V}_{\bmod }^{j}$. The corresponding luminosity function, $\phi_{\text {mod }}$ is shown in black in the top panel of Fig. 5. 
The analysis of the three estimators studied here reveals a similar trend of the corresponding luminosity functions for the faint region, i.e. $M_{\text {bol }} \gtrsim 15 \mathrm{mag}$, being this fact a consequence of a reasonable completeness $(\sim 60 \%)$ for this low-luminosity region. On the contrary, the brighter region suffers from a larger degree of incompleteness, mainly due to a low number statistic. In this situation, the $1 / \mathcal{V}_{\max }$ is markedly inefficient at recovering the original distribution, greatly underestimating the brightest bins. Given that our modified estimator is the only one that properly takes into account the different sources of incompleteness, in what follows we will use the luminosity function derived from it as representative of the halo white dwarf population.

\subsection{The halo white dwarf luminosity function}

In Figure 6 (left panel) we plot the luminosity function derived in this work (black) compared to some of the most recent white dwarf luminosity functions obtained for a high speed or equivalently halo population. In particular, we show the spheroid white dwarf luminosity function obtained from the SuperCOSMOS Sky Survey via the effective volume technique from Rowell \& Hambly (2011) -blue squares-; the luminosity function for high speed white dwarfs, $200<v_{\text {tan }}<500 \mathrm{~km} \mathrm{~s}^{-1}$, derived from SDSS deep proper motion survey by Munn et al. (2016) - green squares -; and the most recent high speed white dwarf luminosity function derived by Lam et al. (2018) from the Pan-STARRS $13 \pi$ Steradian Survey - magenta squares.

First of all, the space density previously derived in Torres et al. (2019a) for our halo candidate sample, $(4.8 \pm 0.4) \times 10^{-5} \mathrm{pc}^{-3}$, is in agreement with the value reported by Lam et al. (2018), (5.291 \pm $0.2) \times 10^{-5} \mathrm{pc}^{-3}$. Moreover, our space density estimate is slightly larger than that of Munn et al. (2016), $(3.5 \pm 0.7) \times 10^{-5} \mathrm{pc}^{-3}$, but it is well below the upper limit of $\left(1.9 \times 10^{-4} \mathrm{pc}^{-3}\right.$ presented by Rowell \& Hambly (2011).

Second, our white dwarf luminosity function is defined in fewer magnitude bins than the rest of samples. That paucity in the number of objects is a consequence of the relative small size of our sample, which is limited within $100 \mathrm{pc}$. However, we need to recall that our sample has been extracted from a nearly volume limited - and thus practically complete - sample, while the rest of samples are magnitude-limited (see Section 2.2 for a detailed analysis of the completeness of our sample). Consequently some biases are expected, in particular for the dimmer intervals of the luminosity function. In this sense, our luminosity function shows a clear peak at $M_{\text {bol }} \sim 15.5$ mag and a marked drop-off for the fainter bins. The location of the peak agrees with the one presented by the Lam et al. (2018)'s luminosity function. At the same time, the slight depression shown at $M_{\text {bol }} \sim 14.5$ mags by our luminosity function is also present in that of Rowell \& Hambly (2011) but seems not to appear in any of the other two distributions. However, the drop-off beyond 15.5 mags is the most relevant feature of our luminosity function, which is not present in any other of the distributions. The only white dwarf luminosity function which extends beyond the cut-off is that of Rowell \& Hambly (2011). Unfortunately, their faintest bins are poorly constrained and no cut-off is observed. Based on the completeness analysis of our sample (see Section 2.2), we can claim that this drop-off is real and thus we are observing for the first time the cut-off of the halo white dwarf luminosity function.

Fitting the cut-off of the white dwarf luminosity function has been extensively used as a consistent technique for estimating ages (e.g García-Berro \& Oswalt 2016). For this purpose we build a set of synthetic white dwarf luminosity functions derived from a $1 \mathrm{Gyr}$ burst of star formation applied at different ages. Our best fit, that is, the one that best reproduces the cut-off of our luminosity function, corresponds to a burst that happened between 11.0-12.0 Gyr in the past. In the right panel of Fig. 6 we show our results for the best fit model when a population of 80:20, DA to non-DA ratio, is considered (red) and when only DAs are taken into account (blue). Both models are able to correctly fit the peak and the cut-off bins. However, the depression previously commented at $M_{\text {bol }} \sim 14.5$ mags seems not to be reproduced by a burst model. The paucity of objects in that bin prevents us from drawing further conclusion. Finally, the possible effects of non-DA stars (in particular those with He-rich atmospheres) are only apparent for the faintest bins of the luminosity function, but these are beyond our observed sample.

\subsection{The mass and age distributions and the star formation history}

The photometric method outlined in Section 2.1 has also allowed us to derive some other important stellar parameters such as the mass and the age of the white dwarfs. In Figure 7 we show the mass distribution (left panel) for our halo white dwarf sample. The mean mass $\left\langle M_{\mathrm{WD}}\right\rangle=0.589 M_{\odot}$ is smaller than the mean value of $\sim 0.65$ generally reported for the single white dwarf disk population (e.g. Tremblay et al. 2016; Bergeron et al. 2019; McCleery et al. 2020). The majority of white dwarfs $(71 \%)$ present a mass smaller than $0.6 M_{\odot}$, which is expected for an old population, as more lowmass progenitors have had enough time to evolve and become white dwarfs of slightly lower masses than the canonical value. On the other hand, we have found just two massive white dwarfs (J1045+5904 and $\mathrm{J} 1123+5742)$ with masses $1.074 \pm 0.033 M_{\odot}$ and $0.893 \pm 0.100 M_{\odot}$, respectively.

In the right panel of Fig. 7 we show the age distribution for our sample of white dwarfs. We recall that the age represented corresponds to the total age of the white dwarfs, that is, the cooling time plus the progenitor lifetime. A first glance to the age distribution reveals that the vast majority of stars $(87 \%)$ have total ages between 8 to $12 \mathrm{Gyr}$. In particular, most of them between $10-12 \mathrm{Gyr}$, being compatible with the 11-12 Gyr burst we applied for fitting the cut-off of the luminosity function (See section 2.3). Three objects found in our sample (J0045-3329, J1049-7559 and J1312-4728) are older than $12 \mathrm{Gyr}$, being the last of them the oldest with an age of $12.41 \pm 0.22$ Gyr. These stars appear older than some of the previously published oldest white dwarfs in the Solar neighborhood. For instance, the objects SDSS J1102+4113 and WD 0346+246 have, respectively, age estimates of 11 and $11.5 \mathrm{Gyr}$ (Kilic et al. 2012). It is worth mentioning that this last object, WD $0346+246$ - which was firstly discover by Hambly et al. (1997) and extensively studied by e.g. Oppenheimer et al. (2001); Bergeron (2001); Kilic et al. (2012) - is also present in our sample with an estimate total age of $11.49 \pm 1.51 \mathrm{Gyr}$ that is in perfect agreement with these previous works.

On the other hand, 12 white dwarfs have age estimates younger than 7 Gyr. In principle, they may be ruled out as genuine halo members but, at the same time, their high speed kinematics are indicative that their origin is different from the bulk of the disk population. They represent $13 \%$ of the sample, a value that can be considered as a general estimate of the contamination of any possible halo white dwarf sample. Moreover, these objects seemed to be clustered at around 2.5 and $5 \mathrm{Gyr}$, respectively. With the aid of our Monte Carlo simulator, we analyze the statistical significance of this apparent clustering. We uniformly distribute 12 objects in the range $0-8 \mathrm{Gyr}$ in intervals of 0.5 Gyr. The probability of obtaining a peak (of three or more stars) is rather high, 0.42 . However, the probability of recovering two of 

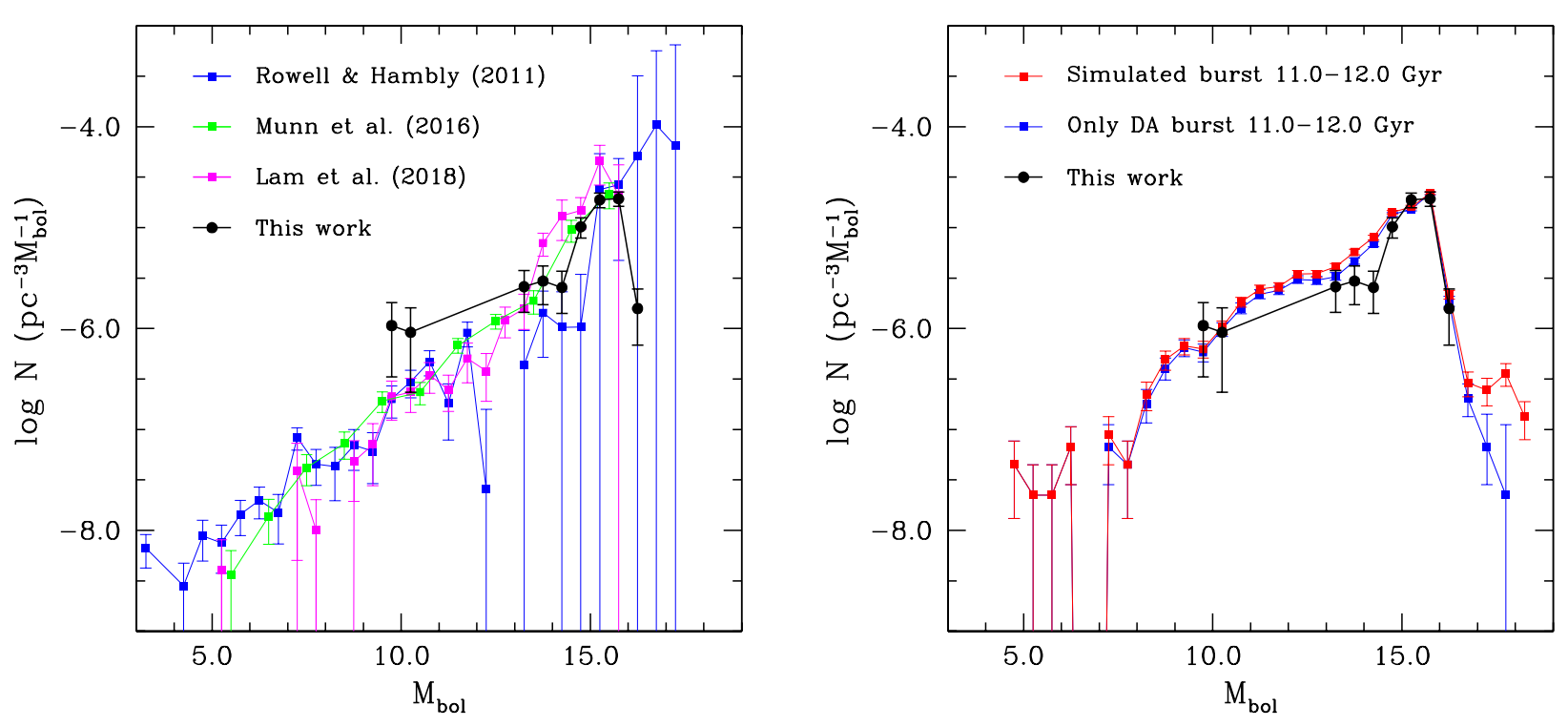

Figure 6. Left panel: Gaia halo white dwarf luminosity function within $100 \mathrm{pc}$ obtained in this work (black dots and lines) compared to the Rowell \& Hambly (2011) spheroid white dwarf luminosity function (blue squares and lines), and the luminosity function for high speed white dwarfs obtained by Munn et al. (2016) (green squares and lines) and Lam et al. (2018) (magenta squares and lines). Right panel: Gaia halo white dwarf luminosity function obtained in this work (black dots and lines), compared with the synthetic populations simulated in this work that best fit the observed features. This is, synthetic populations considering a star formation burst from 11 to 12 Gyrs ago, and a standard proportion of DA to DB white dwarfs of 80-20\% (red squares and lines) and one in which all synthetic white dwarfs are DA (blue squares and lines).

these peaks is considerably low, 0.05. In other words, the observed distribution has a statistical significance of $2 \sigma$ of rejecting that they come from a uniform distribution. In the forthcoming analysis some hypothesis about their origin are presented.

A subproduct of our photometric procedure is the mass of the white dwarf progenitor. Given that we also know the age distribution, we can easily estimate the star formation rate, $\Psi$, as the quantity of stellar mass produced per unit time and unit volume. In Figure 8 we show as a gray histogram our SFR thus computed in units of $M_{\odot} / \mathrm{Gyr} / \mathrm{pc}^{3}$. The SFR resembles, as logically expected, the age distribution previously shown in Fig. 7, although the peaks, in particular for the younger bins, are more pronounced. We observe that the peak of the star formation history is centered at around $11 \mathrm{Gyr}$, which is compatible with the current age of the Gaia-Enceladus encounter. It is worth noting here that our estimate of the halo SFR is restricted to the mass range of those progenitors that, according to our population synthesis model, are able to form a white dwarf, i.e., masses in the range $\sim 1-5.5 M_{\odot}$.

For a comparative purpose we also show in Fig. 8 the recent star formation history from Ruiz-Lara et al. (2020) corresponding to the thin disk (magenta line) and thick disk (blue line) of our Galaxy. In their work, Ruiz-Lara et al. (2020) thoroughly analyzed the HRdiagram derived from the precise astrometric and photometric data provided by Gaia DR2. They found clear evidences that the close encounter of the Milky Way with Saggitarius dwarf galaxy has enhanced the star formation rate at epochs 5.7,1.9 and $1 \mathrm{Gyr}$ in the past. The location of the two older peaks seems to resemble the location of the peaks for the younger white dwarfs found in our sample at 5 and 2.5 Gyr. It can be expected that the close encounter with the Saggitarius dwarf galaxy may have an effect not only in the enhancement of star formation but also in heating the kinematics of the affected stars. Consequently, it is reasonable to link the origin of these high-speed and young white dwarfs to the gravitational effects induced by the pass of the Saggitarius galaxy.
Following the analysis by Ruiz-Lara et al. (2020) we also found an enhancement in the star formation of the thick disk population centered at $\sim 10.5$ Gyr. This enhancement is not associated to the encounters with the Saggitarius dwarf galaxy, but rather to the formation of the thick disk itself. However, this issue is under an intense debate, since it has been also found that in the current scenario of the Milky Way, the formation of the inner stellar halo seems to be triggered by the major merger collision with the Gaia-Enceladus Galaxy (Helmi et al. 2018). In this sense, it has been hypothesized that part of the halo may be associated to the Gaia-Enceladus encounter, and part to an in situ halo (e.g. Gallart et al. 2019). It is beyond of the scope of the present work to unravel the origin of the local halo white dwarf population. Be that as it may, the formation history found for the oldest white dwarfs of our sample is in agreement with this enhacement of the star formation occurred $\simeq 11-12 \mathrm{Gyr}$ in the past.

Nevertheless, the hypothesis above mentioned of the origin of these young halo white dwarfs does not exclude other possible scenarios. For instance, that is the case of the well known LP93-21 white dwarf, which is also present in our sample as J1045+4509. Firstly discovered in the high-proper motion Luyten Palomar survey (Luyten 1968), this white dwarf has been found to have a hot DQ atmosphere, a mass in the range $1.029-1.10 M_{\odot}$ and cooling age estimate of $2.28-2.81 \mathrm{Gyr}$ (Kawka et al. 2020, and references therein). It is worth noting that these values are in perfect in agreement with our estimates of $1.074 \pm 0.033 M_{\odot}$ for the mass and $2.69 \pm 0.25$ for the total age. In a recent paper, Kawka et al. (2020) thoroughly analyze the kinematics of LP 93-21 reaching the conclusion that this DQ white dwarf is the likely merger product of two other white dwarfs, which rejuvenated leading to a shorter cooling age estimate. Furthermore, the kinematics of LP 93-21 is compatible with a dwarf galaxy merger event, suggesting that this white dwarf was either captured by the Milky Way or its orbit was affected by the galaxy merger, hence claiming a total age of $\gtrsim 10 \mathrm{Gyr}$. 

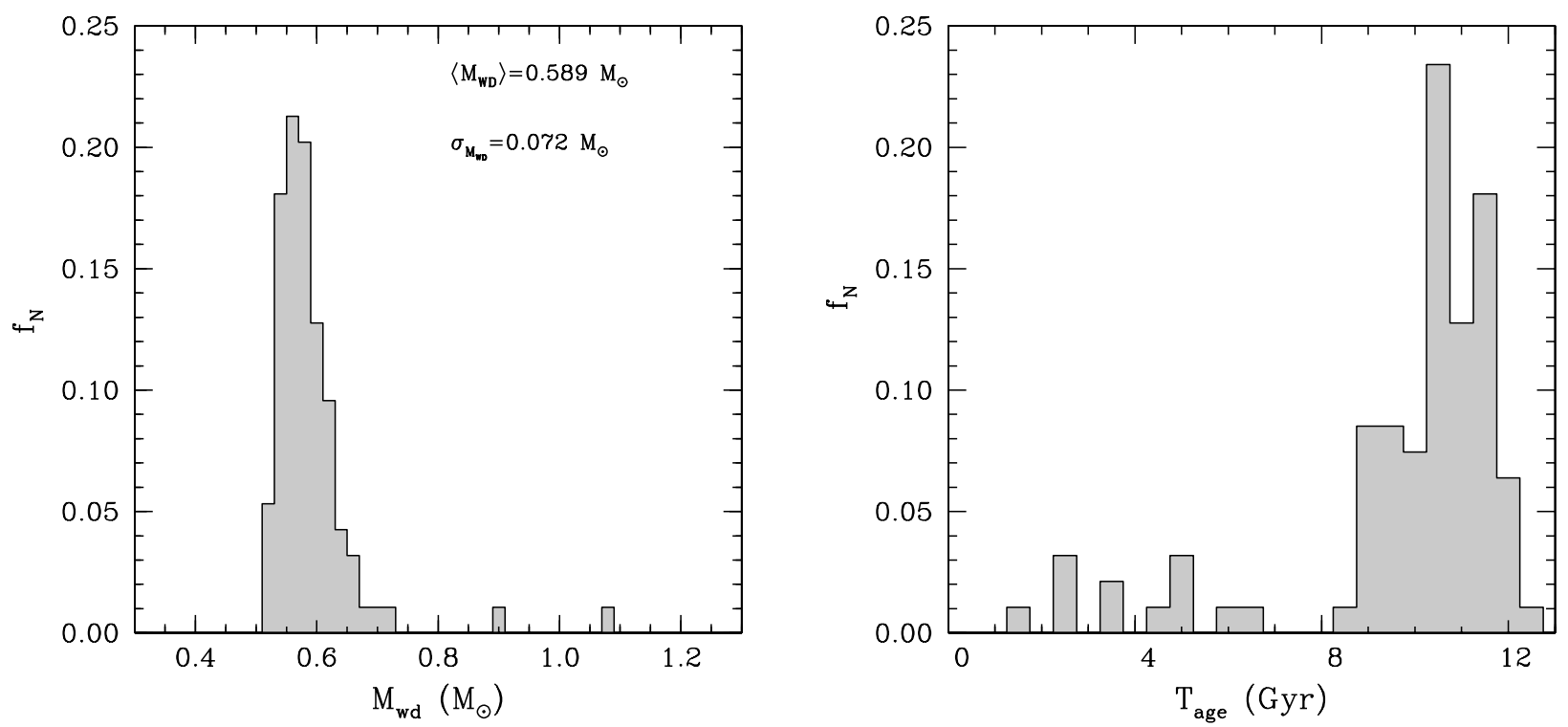

Figure 7. Left panel: Gaia halo white dwarf mass function within $100 \mathrm{pc}$ obtained in this work. Right panel: total age (cooling age plus progenitor lifetime) distribution for our halo white dwarf sample.

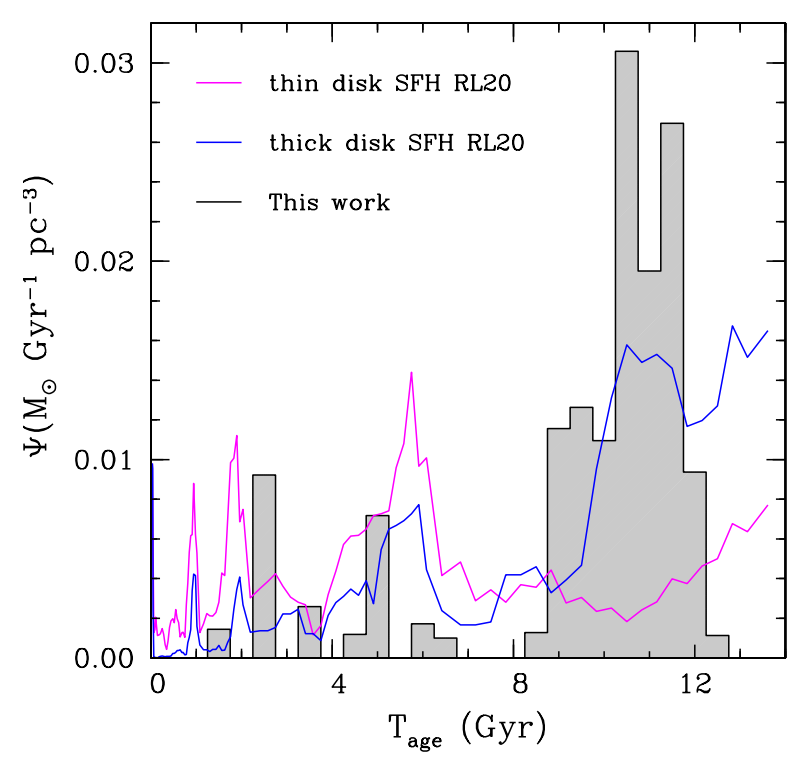

Figure 8. Star formation rate (gray histogram) obtained in this work for the Gaia halo white dwarfs within $100 \mathrm{pc}$. For comparative purposes we also plot the star formation history for the thin disk (magenta line) and thick disk (blue line) population of the Milky Way determined by Ruiz-Lara et al. (2020). These last two distributions are arbitrarily normalized.

On the other hand, we also note that a small fraction of halo white dwarfs could even form as walk-away or runaway secondaries, which are ejected following the disruption of binary systems that underwent either core-collapse or thermonuclear supernova explosions. As an example, Renzo et al. (2019) reported that simulations of systems with primary stars more massive than $7.5 M_{\odot}$, produce ejected mainsequence stars that will eventually become white dwarfs with velocities in the range of $10-90 \mathrm{~km} \mathrm{~s}^{-1}$ (8 percent of simulated binaries).
These ejected main-sequence stars would also be rejuvenated due to mass-transfer occurring before the core-collapse supernova of the companion, hence making the future white dwarf appears younger. Similar or even faster ejection velocities are achieved through the thermonuclear supernova channel (Shen et al. 2018, and refereces therein), where the ejected companions could achieve ejection velocities up to $500 \mathrm{~km} \mathrm{~s}^{-1}$ (main-sequence and helium-core donors) or more than $1000 \mathrm{~km} \mathrm{~s}^{-1}$ (white dwarf donors).

It is beyond the scope of the present work to ascertain the origin of those young halo white dwarf candidates found here. However, along with the possibility that the age distribution of these objects is compatible with the Sagittarius galaxy encounters, an individual analysis is required to fully unravel their origin.

\subsection{Kinematics of the halo white dwarf sample}

The stellar parameter analysis done in the previous sections can be complemented with a kinematic study of the halo white dwarf sample. However, we should be cautious given that only proper motions are provided for the majority of objects of our sample. Consequently, we adopt the standard assumption of null radial velocity in deriving the Galactic components of the velocity $U, V$ and $W$. Despite the biases that this assumption may induce, (e.g. Pauli et al. 2006; Torres et al. 2019a) some of the clustering properties of the sample can remain unaltered (Fuchs \& Dettbarn 2011; Torres et al. 2019b). In this sense, we use the integral of motion space as an appropriate tool for analyzing our sample (e.g Helmi et al. 2006). Since the volume of our sample is relatively small in terms of the size of our Galaxy, we can safely approximate the component of angular momentum perpendicular to the Galactic plane, $L_{z}$, by the $V$-velocity component, as well as the radial and azimuthal Galactic components, $V_{R}$ and $V_{\phi}$, by the components $U$ and $V$, respectively.

In Figure 9 we show the action space corresponding to the integral of motion $V_{\Delta E} \equiv\left(U^{2}+2 V^{2}\right)^{1 / 2}$ as a function of $V$ (left panel) and the Toomre diagram (right panel) for the white dwarfs of our halo candidate sample. For helping the analysis we divided the sam- 

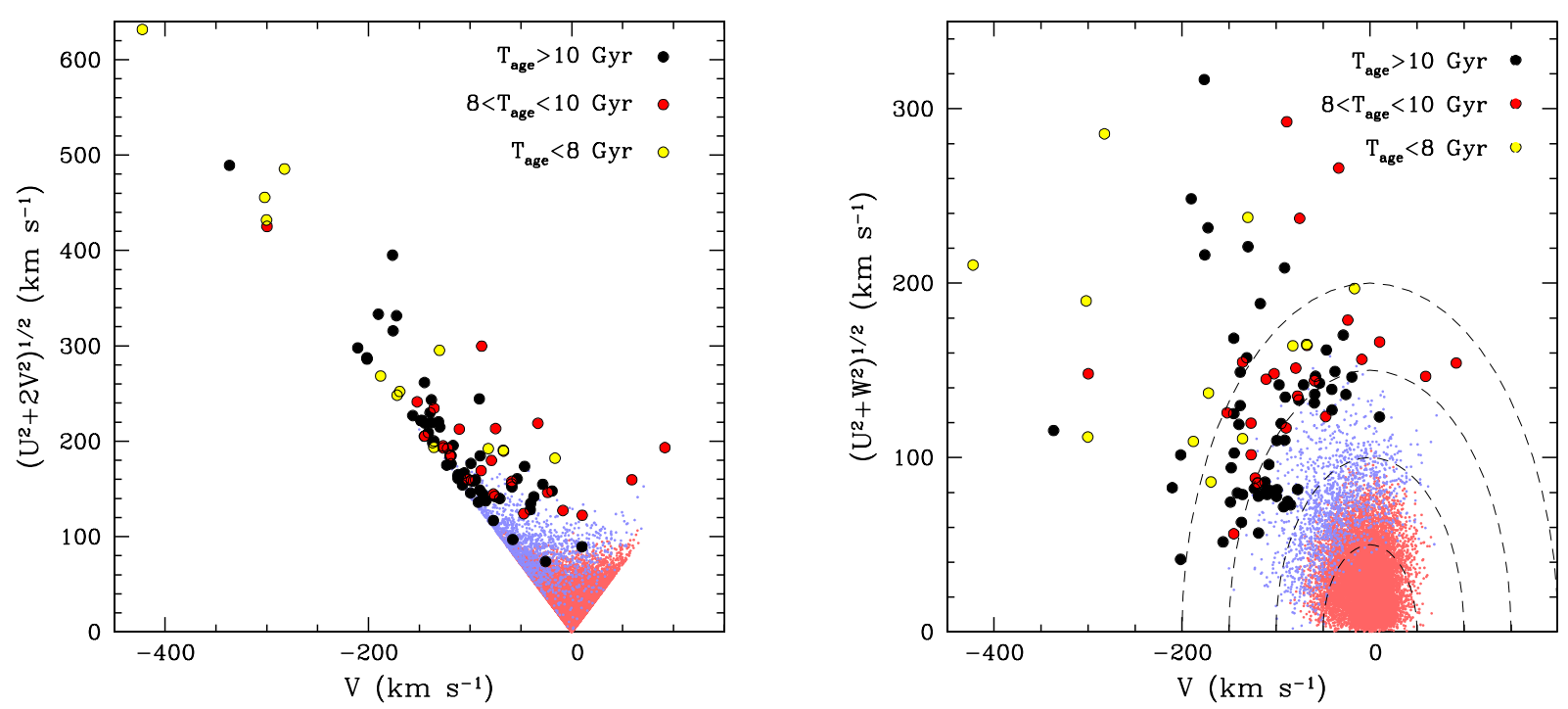

Figure 9. Action space corresponding to the integral of motion $V_{\Delta E} \equiv\left(U^{2}+2 V^{2}\right)^{1 / 2}$ as a function of $V$ (left panel) and the Toomre diagram (right panel) for the white dwarfs of our halo candidate sample (coloured scaled as a function of the age). Also shown, for illustrative purposes, are the thin (light red dots) and thick (light blue dots) velocities for the $100 \mathrm{pc}$ white dwarf sample from Torres et al. (2019a). Dashed lines represent curves for constant $U^{2}+V^{2}+W^{2}$ at values $50,100,150$ and $200 \mathrm{~km} \mathrm{~s}^{-1}$.

ple in three groups attending to the total age: young, $T_{\text {age }}<8 \mathrm{Gyr}$ (yellow dots), middle $8<T_{\text {age }}<10 \mathrm{Gyr}$ (red dots) and old objects $T_{\text {age }}>10 \mathrm{Gyr}$ (black dots). Also shown, for illustrative purposes, are the thin (light red dots) and thick disc (light blue dots) velocities for the $100 \mathrm{pc}$ white dwarf sample from Torres et al. (2019a). The integral of motion $V_{\Delta E}$ can be understood as a measure of the eccentricity, $e$, of the orbit (see Fuchs \& Dettbarn 2011, and references therein). Thus, the left panel shown in Fig. 9 is equivalent to a $\left(L_{z}, e\right)$ diagram. In this sense, we observe that the bulk of old objects of our sample appear with moderately eccentric and retrograde orbits in the range $\approx-100$ to $-200 \mathrm{~km} \mathrm{~s}^{-1}$. A value slightly above that of $\approx-220 \mathrm{~km} \mathrm{~s}^{-1}$ generally adopted for the local standard of rest with respect of the center of the Galaxy, but in agreement for what is considered a classical halo population. On the contrary, those with the highest eccentric and highly retrograde orbits are some of the youngest objects of our sample. These facts suggest to discard these objects as belonging to a typical thick disk population, hence to be thick disk contaminats. Besides, some prograde and relatively middle age objects appear in our sample. However, we should be cautious about this fact, given that one of the effects of the null radial velocity assumption is the misleading prograde orbits, specially for high speed objects, as pointed out in Pauli et al. (2006). Regarding the Toomre diagram (right panel of Fig. 9), a certain clustering seems to be found for the oldest objects of our sample while a greater dispersion is found for the middle and younger ones. In particular, the youngest objects exhibit, on average, the largest speeds.

\section{CONCLUSIONS}

The Gaia space mission on its DR2 has provided an unprecedented wealth of photometric and astrometric data. In particular, the white dwarf population has found to be nearly complete up to $100 \mathrm{pc}$ around the Sun. In this sample, 95 white dwarfs have been identified as belonging to a halo population. In the present work, this subsample has been analyzed and the stellar parameters of the objects has been derived.

Observations made with the Very Large Telescope UT1 equipped with the FORS2 spectrograph allowed us to obtain low-resolution spectra for 27 of our 95 halo white dwarf candidates. By applying a fitting routine to the observed spectra we derive effective temperatures for $24 \mathrm{DCs}$ and $1 \mathrm{DA}$ of the observed sample. We also derived the surface gravity of the DA white dwarf via fitting its Balmer lines, which results also in obtaining its mass, radius and luminosity via interpolating the observed effective temperature and surface gravity in the appropriate cooling sequences. On the other hand, we apply a procedure based on Gaia astro-photometry and on a detailed population synthesis code, that permitted to derive the stellar parameters of the white dwarfs. The parameters thus derived were tested with those available from spectroscopy. The agreement of both methods, in particular in the effective temperature parameter, guarantees us the reliability of our photometric routine to derive the stellar parameters, i.e., mass, radius, bolometric luminosity and age, for the whole sample of 95 halo white dwarf candidates.

The major results found for the different parameter distributions are summarized as follows:

- A generalized version of the modified volume technique, which allow us to take into account all kind of incompleteness sources, has been used to build the $100 \mathrm{pc}$ halo white dwarf luminosity function. The halo luminosity function reasonably presents the first ever detected evidence of a clear cut-off at faint bolometric magnitudes. Although the number of objects of our sample is relatively small compared to other published halo white dwarf samples, the completeness analysis reveals $\sim 60 \%$ completeness for the faint region thus guarantying an acceptable statistical significance of the cut-off found in our sample. The corresponding fitting of the cut-off leads to an age of $\approx 12 \pm 0.5 \mathrm{Gyr}$.

- The halo white dwarf mass distribution peaks at $0.589 M_{\odot}$, 
with most of the stars $(71 \%)$ having masses below $0.6 M_{\odot}$. Only two objects have been found to be more massive than $0.8 M_{\odot}$.

- The majority of white dwarfs $(60 \%)$ have total ages (cooling time plus progenitor lifetime) older than $10 \mathrm{Gyr}$. In particular, we found 3 objects with total ages above 12 Gyr, being the object J13124728 the oldest white dwarf found so far with an age of $12.41 \pm$ $0.22 \mathrm{Gyr}$.

- The star formation history is basically reproduced by a burst of star formation occurring from 10 to $12 \mathrm{Gyr}$ and extended up to $8 \mathrm{Gyr}$. The peak of the star formation history is centered at around $11 \mathrm{Gyr}$, which is compatible with the current age of the Gaia-Enceladus encounter.

- $13 \%$ of our halo sample is contaminated by high-speed young objects (total age $<7 \mathrm{Gyr}$ ). The origin of these white dwarfs is unclear but their age distribution may be compatible with the pass of the Sagittarius galaxy. An individual analysis is required to unravel the origin of each object.

- Finally, the kinematics analysis of the halo white dwarf sample reveals that there is some clustering of the oldest $\left(T_{\mathrm{age}}>10 \mathrm{Gyr}\right)$ objects of the sample suggesting a common origin, whereas on the contrary, youngest objects exhibit larger eccentric and retrograde orbits. Some prograde orbits have also been found, however, the lack of radial velocity observations prevents us to obtain definitive conclusions.

In this work we have demonstrated the utility of white dwarfs to address important open questions in astronomy such as the age of the Galactic halo and its star formation history. These objects can also potentially help to reveal the past history and evolution of our Galaxy, an issue that we will analyse in the future with the forthcoming data releases of Gaia.

\section{ACKNOWLEDGEMENTS}

We wish to thank the suggestions and comments of our anonymous referee that strongly improved the original version of this paper. This work was partially supported by the MINECO grant AYA2017-86274-P and the Ramón y Cajal programme RYC-2016-20254 and by the AGAUR (SGR-661/2017). ST wants to thank Marco Lam for kindly providing the set of luminosity functions used in this work. ST also wants to thank Carme Gallart for useful discussions on the star formation history of the Galaxy. This work has made use of data from the European Space Agency (ESA) mission Gaia (https: //www. cosmos.esa.int/gaia), processed by the Gaia Data Processing and Analysis Consortium (DPAC, https://www. cosmos. esa.int/web/gaia/dpac/consortium). Funding for the DPAC has been provided by national institutions, in particular the institutions participating in the Gaia Multilateral Agreement.

Based on observations collected at the European Southern Observatory under ESO programme(s) 60.A-9203(E) and 0103.D0271(A,B,C,D,E).

RR has received funding from the postdoctoral fellowship programme Beatriu de Pinós, funded by the Secretary of Universities and Research (Government of Catalonia) and by the Horizon 2020 programme of research and innovation of the European Union under the Maria Skłodowska-Curie grant agreement No 801370.

\section{DATA AVAILABILITY STATEMENT}

The data underlying this article are available in the article. Supplementary material will be shared on reasonable request to the corresponding author.

\section{REFERENCES}

Alcock C., et al., 2000, The Astrophysical Journal, 542, 281

Althaus L. G., Córsico A. H., Isern J., García-Berro E., 2010, A\&ARv, 18,471

Althaus L. G., Camisassa M. E., Miller Bertolami M. M., Córsico A. H., García-Berro E., 2015, A\&A, 576, A9

Appenzeller I., et al., 1998, The Messenger, 94, 1

Bergeron P., 2001, ApJ, 558, 369

Bergeron P., Ruiz M. T., Hamuy M., Leggett S. K., Currie M. J., Lajoie C.-P., Dufour P., 2005, ApJ, 625, 838

Bergeron P., Dufour P., Fontaine G., Coutu S., Blouin S., Genest-Beaulieu C., Bédard A., Rolland B., 2019, ApJ, 876, 67

Calamida A., et al., 2014, ApJ, 790, 164

Camisassa M. E., Althaus L. G., Córsico A. H., Vinyoles N., Serenelli A. M., Isern J., Miller Bertolami M. M., García-Berro E., 2016, ApJ, 823, 158

Camisassa M. E., Althaus L. G., Rohrmann R. D., García-Berro E., Torres S., Córsico A. H., Wachlin F. C., 2017, ApJ, 839, 11

Camisassa M. E., et al., 2019, A\&A, 625, A87

Catalán S., Isern J., García-Berro E., Ribas I., Allende Prieto C., Bonanos A. Z., 2008, A\&A, 477, 213

Catalán S., et al., 2012, A\&A, 546, L3

Cojocaru R., Torres S., Althaus L. G., Isern J., García-Berro E., 2015, A\&A, 581, A108

Felten J. E., 1976, ApJ, 207, 700

Flynn C., Holopainen J., Holmberg J., 2003, Monthly Notices of the Royal Astronomical Society, 339, 817

Fuchs B., Dettbarn C., 2011, AJ, 141, 5

Gaia Collaboration et al., 2018, A\&A, 616, A1

Gaia Collaboration et al., 2020, arXiv e-prints, p. arXiv:2012.02061

Gallart C., Bernard E. J., Brook C. B., Ruiz-Lara T., Cassisi S., Hill V., Monelli M., 2019, Nature Astronomy, 3, 932

García-Berro E., Oswalt T. D., 2016, New Astron. Rev., 72, 1

García-Berro E. Torres S., Isern J., Burkert A., 1999, MNRAS, 302, 173

García-Berro E., Torres S., Isern J., Burkert A., 2004, A\&A, 418, 53

García-Berro E., et al., 2010, Nature, 465, 194

Geijo E. M., Torres S., Isern J., García-Berro E., 2006, MNRAS, 369, 1654

Gentile Fusillo N. P., et al., 2019, MNRAS, 482, 4570

Gianninas A., Curd B., Thorstensen J. R., Kilic M., Bergeron P., Andrews J. J., Canton P., Agüeros M. A., 2015, MNRAS, 449, 3966

Hall P. B., Kowalski P. M., Harris H. C., Awal A., Leggett S. K., Kilic M., Anderson S. F., Gates E., 2008, AJ, 136, 76

Hambly N. C., Smartt S. J., Hodgkin S. T., 1997, ApJ, 489, L157

Hansen B. M. S., et al., 2013, Nature, 500, 51

Helmi A., Navarro J. F., Nordström B., Holmberg J., Abadi M. G., Steinmetz M., 2006, Monthly Notices of the Royal Astronomical Society, 365,1309

Helmi A., Babusiaux C., Koppelman H. H., Massari D., Veljanoski J., Brown A. G. A., 2018, Nature, 563,85

Hidalgo S., et al., 2018, The Astrophysical Journal, 856

Ibata R., Irwin M., Bienaymé O., Scholz R., Guibert J., 2000, ApJ, 532, L41

Isern J., García-Berro E., Hernanz M., Mochkovitch R., Torres S., 1998, ApJ, 503,239

Jeffery E. J., von Hippel T., DeGennaro S., van Dyk D. A., Stein N., Jefferys W. H., 2011, ApJ, 730, 35

Jiménez-Esteban F. M., Torres S., Rebassa-Mansergas A., Skorobogatov G., Solano E., Cantero C., Rodrigo C., 2018, MNRAS, 480, 4505

Kalirai J. S., 2012, Nature, 486, 90

Kawka A., Vennes S., 2012, Monthly Notices of the Royal Astronomical Society, 425, 1394

Kawka A., Vennes S., Ferrario L., 2020, MNRAS, 491, L40 
Kilic M., von Hippel T., Mendez R. A., Winget D. E., 2004, ApJ, 609, 766

Kilic M., Thorstensen J. R., Kowalski P. M., Andrews J., 2012, MNRAS, 423, L132

Kilic M., Munn J. A., Harris H. C., von Hippel T., Liebert J. W., Williams K. A., Jeffery E., DeGennaro S., 2017, ApJ, 837, 162

Kilic M., Bergeron P., Dame K., Hambly N. C., Rowell N., Crawford C. L., 2018, MNRAS,

Koester D., 2010, Mem. Soc. Astron. Italiana, 81, 921

Lam M. C., Rowell N., Hambly N. C., 2015, Monthly Notices of the Royal Astronomical Society, 450, 4098

Lam M. C., et al., 2018, Monthly Notices of the Royal Astronomical Society, 482,715

Liebert J., Dahn C. C., Monet D. G., 1989, in Wegner G., ed., Lecture Notes in Physics, Berlin Springer Verlag Vol. 328, IAU Colloq. 114: White Dwarfs. pp 15-23, doi:10.1007/3-540-51031-1_287

Luyten W. J., 1968, Proper Motion Survey, University of Minnesota, 13, 1

Marsh T. R., 1989, PASP, 101, 1032

McCleery J., et al., 2020, MNRAS, 499, 1890

Mochkovitch R., Garcia-Berro E., Hernanz M., Isern J., Panis J. F., 1990, A\&A, 233, 456

Monet D. G., Fisher M. D., Liebert J., Canzian B., Harris H. C., Reid I. N., 2000, AJ, 120, 1541

Munn J. A., et al., 2016, The Astronomical Journal, 153, 10

Napiwotzki R., et al., 2020, VizieR Online Data Catalog, pp J/A+A/638/A131

Nelson C. A., Cook K. H., Axelrod T. S., Mould J. R., Alcock C., 2002, ApJ, 573,644

Oppenheimer B. R., et al., 2001, ApJ, 550, 448

Pauli E.-M., Napiwotzki R., Heber U., Altmann M., Odenkirchen M., 2006, A\&A, 447, 173

Planck Collaboration et al., 2016, A\&A, 594, A13

Rebassa-Mansergas A., Gänsicke B. T., Rodríguez-Gil P., Schreiber M. R., Koester D., 2007, MNRAS, 382, 1377

Renzo M., et al., 2019, A\&A, 624, A66

Rowell N., Hambly N. C., 2011, MNRAS, 417, 93

Ruiz-Lara T., Gallart C., Bernard E. J., Cassisi S., 2020, Nature Astronomy,

Schmidt M., 1968, ApJ, 151, 393

Shen K. J., et al., 2018, ApJ, 865, 15

Si S., van Dyk D. A., von Hippel T., Robinson E., Webster A., Stenning D., 2017, MNRAS, 468, 4374

Torres S., García-Berro E., 2016, A\&A, 588, A35

Torres S., García-Berro E., Isern J., 1998, The Astrophysical Journal Letters, 508, L71

Torres S., García-Berro E., Burkert A., Isern J., 2001, MNRAS, 328, 492

Torres S., García-Berro E., Burkert A., Isern J., 2002, MNRAS, 336, 971

Torres S., García-Berro E., Isern J., Figueras F., 2005, MNRAS, 360, 1381

Torres S., García-Berro E., Althaus L. G., Camisassa M. E., 2015, A\&A, 581, A90

Torres S., García-Berro E., Cojocaru R., Calamida A., 2018, MNRAS,

Torres S., Cantero C., Rebassa-Mansergas A., Skorobogatov G., JiménezEsteban F. M., Solano E., 2019a, MNRAS, 485, 5573

Torres S., Cantero C., Camisassa M. E., Antoja T., Rebassa-Mansergas A., Althaus L. r. G., Thelemaque T., Cánovas H., 2019b, A\&A, 629, L6

Tremblay P. E., Cummings J., Kalirai J. S., Gänsicke B. T., Gentile-Fusillo N., Raddi R., 2016, MNRAS, 461, 2100

Tremblay P. E., et al., 2020, MNRAS,

This paper has been typeset from a $\mathrm{T}_{\mathrm{E}} \mathrm{X} / \mathrm{LT}_{\mathrm{E}} \mathrm{X}$ file prepared by the author. 